\title{
Phosphorylated AKT preserves stallion sperm viability and motility by inhibiting caspases 3 and 7
}

\author{
Juan M Gallardo Bolaños ${ }^{1}$, Carolina M Balao da Silva ${ }^{1}$, Patricia Martín Muñoz ${ }^{1}$, \\ Antolín Morillo Rodríguez ${ }^{1}$, María Plaza Dávila ${ }^{1}$, Heriberto Rodríguez-Martínez ${ }^{3}$, \\ Inés M Aparicio ${ }^{2}$, José A Tapia ${ }^{2}$, Cristina Ortega Ferrusola ${ }^{1}$ and Fernando J Peña ${ }^{1}$ \\ ${ }^{1}$ Laboratory of Equine Reproduction and Equine Spermatology, Faculty of Veterinary Medicine, Veterinary Teaching \\ Hospital and ${ }^{2}$ Department of Physiology, Faculty of Veterinary Medicine, University of Extremadura, Avd de la \\ Universidad s/n, 10003 Cáceres, Spain and ${ }^{3}$ Department of Clinical and Experimental Medicine, Faculty of \\ Health Sciences, Linköping University, Linköping, Sweden
}

Correspondence should be addressed to FJ Peña; Email: fjuanpvega@unex.es

\begin{abstract}
AKT, also referred to as protein kinase B (PKB or RAC), plays a critical role in controlling cell survival and apoptosis. To gain insights into the mechanisms regulating sperm survival after ejaculation, the role of AKT was investigated in stallion spermatozoa using a specific inhibitor and a phosphoflow approach. Stallion spermatozoa were washed and incubated in Biggers-Whitten-Whittingham medium, supplemented with 1\% polyvinyl alcohol (PVA) in the presence of 0 (vehicle), 10, 20 or $30 \mu \mathrm{M} \mathrm{SH5,} \mathrm{an} \mathrm{AKT} \mathrm{inhibitor.} \mathrm{SH5} \mathrm{treatment}$ reduced the percentage of sperm displaying AKT phosphorylation, with inhibition reaching a maximum after $1 \mathrm{~h}$ of incubation.

This decrease in phosphorylation was attributable to either dephosphorylation or suppression of the active phosphorylation pathway. Stallion spermatozoa spontaneously dephosphorylated during in vitro incubation, resulting in a lack of a difference in AKT phosphorylation between the SH5-treated sperm and the control after $4 \mathrm{~h}$ of incubation. AKT inhibition decreased the proportion of motile spermatozoa (total and progressive) and the sperm velocity. Similarly, AKT inhibition reduced membrane integrity, leading to increased membrane permeability and reduced the mitochondrial membrane potential concomitantly with activation of caspases 3 and 7. However, the percentage of spermatozoa exhibiting oxidative stress, the production of mitochondrial superoxide radicals, DNA oxidation and DNA fragmentation were not affected by AKT inhibition. It is concluded that AKT maintains the membrane integrity of ejaculated stallion spermatozoa, presumably by inhibiting caspases 3 and 7 , which prevents the progression of spermatozoa to an incomplete form of apoptosis.
\end{abstract}

\section{Free Spanish abstract}

A Spanish translation of this abstract is freely available at http://www.reproduction-online.org/content/148/2/221/suppl/DC1. Reproduction (2014) 148 221-235

\section{Introduction}

Artificial insemination with chilled transported semen is widely used in the equine industry, with most breeding registries now accepting this reproductive technology (Peña et al. 2012). However, the viability and fertilisation capacity of cooled stallion spermatozoa are typically maintained for only a few days or, in some cases, only several hours (Macías Garcia et al. 2012). Apoptosis is a form of programmed cell death that is executed by caspases, a family of cysteine proteases that are activated through two major pathways. Caspases are intrinsically activated by cellular stress through the liberation of mitochondrial pro-apoptotic proteins that activate caspase 9. Caspases are also extrinsically activated by ligand-activated death receptors that induce cleavage of caspase 8 (Aitken et al. 2011). The occurrence of apoptosis in ejaculated spermatozoa remains under debate. The theory of abortive apoptosis considers spermatozoal apoptosis as a remnant from spermatogenesis (Sakkas et al. 2002) on the basis that spermatozoa are terminal, transcriptionally silent cells. This theory is contradicted by findings that biotechnological procedures such as cooling, storage and cryopreservation induce features typical of apoptosis in ejaculated spermatozoa (Moran et al. 2008, OrtegaFerrusola et al. 2008, Ortega Ferrusola et al. 2009a,b, Said et al. 2010, Aitken et al. 2012). Thus, the apoptosis phenomenon can be triggered in spermatozoa after ejaculation through the intrinsic apoptotic pathway (Peña et al. 2003a). Determining whether apoptosis occurs in ejaculated spermatozoa may have substantial 
implications for human and animal-assistive reproductive biotechnologies where sperm are manipulated for extended periods of time (Peña et al. 2006, Ortega-Ferrusola et al. 2009c).

AKT, also referred to as protein kinase B (PKB or RAC), plays a critical role in controlling survival and apoptosis in somatic cells (Hers et al. 2011). Growing evidence suggests that AKT is also involved in the regulation of sperm motility and survival (Aparicio et al. 2005, Aquila et al. 2007, Koppers et al. 2011, Parte et al. 2012). Although the first component of this pathway, phosphatidylinositide 3-kinase (PIK3CA (PI3K)), has been the subject of several studies in sperm biology, AKT has received insufficient attention despite recent research identifying it as a key factor for sperm survival after ejaculation. AKT suppresses sperm capacitation and prevents spermatozoa from entering the apoptotic cascade that leads to sperm senescence and death (Pujianto et al. 2010). We recently demonstrated that after cryopreservation (Ortega-Ferrusola et al. 2008, 2010) and chilling (Macías Garcia et al. 2012), stallion sperm death occurs through apoptosis-related phenomena. In view of these findings and to determine the role of AKT in regulating the survival of ejaculated stallion spermatozoa, we investigated stallion sperm survival using a specific inhibitor and a phosphoflow cytometry (flow cytometry-based approaches to measure changes in the phosphorylation status of key intracellular signalling molecules, at a single-cell level) approach involving antibodies against phosphorylated forms of a particular protein (Tazzari et al. 2002, Krutzik et al. 2004, Schulz et al. 2007, 2012). Our results indicate that survival of ejaculated spermatozoa is regulated by AKT through the inhibition of an apoptosis-like mechanism. This mechanism, previously proposed in humans (Aitken et al. 2011), may result in the silent removal of defective spermatozoa from the female genitalia (Lessig et al. 2007). If a similar mechanism is conserved in horses, it may be possible to use equine spermatozoa to model human spermatozoa, particularly in areas that are less explored in humans, such as sperm defects and preservation. Moreover, we demonstrate the utility of the phosphoflow approach for studying sperm biology.

\section{Material and methods}

\section{Reagents and media}

Ethidium homodimer (Eth), 5, 5' ,6,6'-tetrachloro-1, 1',3,3' tetraethyl benzymidazolyl carbocianyne iodine (JC-1), Mitrotracker Deep Red, YO-PRO-1, CellEvent caspases 3 and 7 detection reagent, CellROX Deep Red, MitoSox Red, Sytox Green and the TUNEL assay were purchased from Molecular Probes (Leiden, The Netherlands). The AKT inhibitor SH5 and the OxyDNA Assay Kit were purchased from Calbiochem (San Diego, CA, USA). The AKT1/2 kinase inhibitors carbenexolone and probenecid were purchased from Sigma. Anti-pannexin-1 was purchased from Abcam (Cambridge, UK). Anti-phospho-
AKT $\left(\mathrm{pSer}^{473}\right)$ and anti-phospho-AKT $\left(\mathrm{pThr}^{308}\right)$ Alexa Flour 488- and Alexa Flour 647-conjugated MABs and IgG XP Isotype Controls (Alexa Fluor 488 and Alexa Flour 647 conjugates) were purchased from Cell Signalling Technology (Danvers, MA, USA).

\section{Semen collection and processing}

Semen was obtained from seven Pure Spanish horses (PRE; three ejaculates each) that were individually housed at the Veterinary Teaching Hospital of the University of Extremadura (Cáceres, Spain). Stallions were maintained according to institutional and European regulations. Ejaculates were collected on a regular basis (two collections per week) during the 2012 breeding season, using a pre-warmed, lubricated Missouri model artificial vagina with an inline filter to eliminate the gel fraction. The semen samples were immediately transported to the laboratory for evaluation and processing. The ejaculate samples were diluted 1:2 in INRA96 (IMV, L'Aigle, France) centrifuged $(600 \mathrm{~g}, 10 \mathrm{~min})$ and re-suspended in Biggers-Whitten-Whittingham (BWW; Macías Garcia et al 2011a,b) medium supplemented with $1 \%$ polyvinyl alcohol (PVA) to obtain a concentration of $50 \times 10^{6}$ spermatozoa $/ \mathrm{ml}$. All experiments followed a split sample design with every ejaculate sample divided between the control and the treatment groups.

To study the role of AKT in the maintenance of stallion sperm motility and viability, split samples of stallion spermatozoa were incubated in BWW medium in the presence of a specific inhibitor of AKT (D-3-deoxy-2-O-methyl-myo-inositol-1-((R)-2methoxy-3-(octadecyloxy) propyl hydrogen phosphate) ( $\mathrm{SH}-5$ ) (Sethi et al. 2008) (0 (vehicle), 10, 20 and $30 \mu \mathrm{M}$ in DMSO) for up to $6 \mathrm{~h}$ at $37^{\circ} \mathrm{C}$ in BWW medium supplemented with $1 \%$ PVA. The final concentration of DMSO in all samples was $0.01 \%$. SH5 is a cell permeable, reversible, substratecompetitive phosphatidylinositol analogue that inhibits AKT activation and the activation of select downstream substrates without decreasing phosphorylation of PDK-1 or other kinases downstream of RAS, such as MAPK. SH5 has been widely used as specific inhibitor of AKT in many cell lines (Lan et al. 2011, Busch et al. 2012, Qiao et al. 2013, Yang et al. 2013), acting as a potent inducer of apoptosis that selectively kills various types of cancer cells that contain high levels of active AKT. We used a second inhibitor, a AKT1/2 inhibitor, in additional experiments (Gilot et al. 2010). The AKT phosphorylation status was monitored by flow cytometry. All experiments were repeated using three different ejaculates from each of the seven stallions (21 replicates in total).

\section{Western blotting}

Stallion semen was centrifuged and washed twice with PBS. After washing, the sperm cells were sonicated for $5 \mathrm{~s}$ at $4{ }^{\circ} \mathrm{C}$ in $100 \mu \mathrm{l}$ of lysis buffer consisting of $50 \mathrm{mM}$ Tris/ $\mathrm{HCl}, 150 \mathrm{mM}$ $\mathrm{NaCl}, 1 \%$ Triton $\mathrm{X}-100,1 \%$ deoxycholate, $1 \mathrm{mM}$ EGTA, $0.4 \mathrm{mM}$ EDTA, a protease inhibitor cocktail (Complete, EDTA-free, cat no. 11836170001, Roche Diagnostics $\mathrm{GmbH}$ ) and $0.2 \mathrm{mM} \mathrm{Na}_{3} \mathrm{VO}_{4}, \mathrm{pH}$ 7.5. The homogenates were clarified by centrifugation at $10000 \mathrm{~g}\left(15 \mathrm{~min}, 4{ }^{\circ} \mathrm{C}\right)$, and the 
supernatants were used for protein analysis after dilution with $4 \times$ SDS sample buffer. Total protein samples $(25 \mu \mathrm{g} /$ well $)$ from stallion sperm were fractionated by SDS-PAGE using 4-20\% polyacrylamide gradient gels and transferred to nitrocellulose membranes. After blocking, the membranes were incubated overnight at $4{ }^{\circ} \mathrm{C}$ with anti-pannexin-1 (1:1000), anti-pAKT $\operatorname{Ser}^{473}$ (1:1000) and anti-pAKT $\operatorname{Thr}^{308}$ (1:1000). On the following day, the membranes were washed twice and incubated for $45 \mathrm{~min}$ at $25^{\circ} \mathrm{C}$ with anti-rabbit IgG HRPconjugated secondary antibody. The membranes were then washed again, incubated with ECL detection reagents and finally were used to expose Hyperfilm ECL Films (Amersham). The positive controls for pAKT $\mathrm{Ser}^{473}$ and $\mathrm{Thr}^{308}$ were pancreatic acini lysates. The positive controls for pannexin-1 were brain lysates. The intensity and molecular weight of the bands were quantified using Scion Image Software for Windows, version 4.02 (Scion Corp., Frederick, MD, USA) and normalised against the values for $\beta$-actin.

\section{Immunocytochemistry}

Spermatozoa were washed and suspended in PBS, with an adjustment of the cell concentration to $1 \times 10^{6}$ cells $/ \mathrm{ml}$. Fifteen microlitres of the sperm suspension were spread on poly-Llysine-coated slides and allowed to attach for $10 \mathrm{~min}$. The cells were then fixed with 3\% formaldehyde in PBS for 15 min and permeabilised with 0.2 Triton X-100 in PBS for 5 min. Slides were washed three times for $10 \mathrm{~min}$ each with PBS and incubated in PBS supplemented with 5\% BSA (w/v) for $90 \mathrm{~min}$ to block non-specific sites. After blocking, the slides were incubated overnight at $4{ }^{\circ} \mathrm{C}$ with anti-phospho-AKT Ser ${ }^{473}$ (1:50) diluted in PBS containing 5\% BSA (w/v). On the next day, samples were extensively washed with PBS and further incubated with an Alexa 488-conjugated goat anti-rabbit antibody for $45 \mathrm{~min}$ at room temperature (RT). Finally, the slides were washed with PBS and examined with a Bio-Rad MRC1024 confocal microscope with a $60 \times$ objective in oil immersion. The samples were excited at $488 \mathrm{~nm}$ with an argon laser and emission was recorded using a $515 \mathrm{~nm}$ long-pass filter set. Samples without any primary antibody were assessed to confirm the absence of non-specific staining.

\section{Sperm motility}

Sperm motility and kinematics were assessed using a CASA system (ISAS Proiser, Valencia, Spain; Peña et al. 2005a, Nuñez-Martinez et al. 2007a, Gonzalez-Fernandez et al. 2009). Semen was loaded in a Leja chamber with a depth of $20 \mu \mathrm{m}$ (Leja, Amsterdam, The Netherlands) and placed on a warmed $\left(37^{\circ} \mathrm{C}\right)$ stage. The analysis was based on an examination of 60 consecutive digitalised images obtained from each field using a $10 \times$ negative phase-contrast objective. At least three different fields were recorded to ensure that at least 200 spermatozoa were analysed per sample. Images were taken at 1-s intervals. Objects incorrectly identified as spermatozoa were excluded by observation using the playback function. Spermatozoa with a VAP of $<15 \mu \mathrm{m} / \mathrm{s}$ were considered immotile, whereas spermatozoa with a VAP $>15 \mu \mathrm{m} / \mathrm{s}$ were considered motile. Spermatozoa deviating
$<45 \%$ from a straight line were designated as linearly motile and spermatozoa with a circular velocity $(\mathrm{VCL})>45 \mu \mathrm{m} / \mathrm{s}$ were designated as rapid sperm. The absolute and re-calculated kinematic parameters measured by CASA included the following (Martínez-Pastor et al; 2011): curvilinear velocity, the time-average velocity of the sperm head along its actual trajectory $(\mathrm{VCL}(\mu \mathrm{m} / \mathrm{s}))$; straight line velocity, the time average velocity of the sperm head along a straight line from its firstdetected position to its last-detected position (VSL $(\mu \mathrm{m} / \mathrm{s}))$ and average path velocity, the time average velocity of the sperm head along its average trajectory. The average trajectory is computed by smoothing the average trajectory $(\operatorname{VAP}(\mu \mathrm{m} / \mathrm{s}))$.

\section{Flow cytometry}

Flow cytometric analyses were conducted using a MACSQuant Analyser 10 (Miltenyi Biotech, Madrid, Spain) flow cytometer, equipped with three lasers emitting at 405, 488 and $635 \mathrm{~nm}$ and ten photomultiplier tubes (V1 (excitation $405 \mathrm{~nm}$ and emission 450/50 nm), V2 (excitation $405 \mathrm{~nm}$ and emission 525/50 nm), B1 (excitation $488 \mathrm{~nm}$ and emission 525/50 nm), B2 (excitation $488 \mathrm{~nm}$ and emission 585/40 nm), B3 (excitation $488 \mathrm{~nm}$ and emission $655-730 \mathrm{~nm}(655 \mathrm{LP}+$ split 730)), B4 (excitation $488 \mathrm{~nm}$ and emission $750 \mathrm{LP}), \mathrm{R} 1$ (excitation $635 \mathrm{~nm}$ and emission 655-730 nm (655LP+split 730)) and R2 (excitation $635 \mathrm{~nm}$ and emission filter 750 LP)). The system was controlled using MACSQuantify Software. Sperm subpopulations were divided by quadrants to quantify the frequency of each subpopulation. Forward and sideways light scatter were recorded for a total of 50000 events/sample. Nonsperm events were eliminated by gating the sperm population after Hoechst 33342 staining. All positive events for Hoechst 33342 within a certain range of the side scatter (SCC) distribution were considered spermatozoa. The instrument was calibrated daily using specific calibration beads provided by the manufacturer, and compensation of spectral overlap was performed before each experiment. Compensation was performed before each particular experiment using negative (unstained) and positive (single stained) controls for each single-stained compensation control sample following published recommendations for multicolour flow cytometry (Baumgarth \& Roederer 2000, Roederer 2001) and the instructions in the MacsQuant Instrument Software guide (Miltenyi Biotech). Also thresholds for each quadrant were determined using unstained, isotype controls and singlestained control samples for each of the probes used in each individual experiment.

\section{Phosphoflow detection of phosphorylated AKT $\left(\mathrm{Ser}^{473}\right)$ and $A K T\left(T h r^{308}\right)$ in stallion spermatozoa}

The spermatozoa $\left(1 \times 10^{6} / \mathrm{ml}\right)$ were washed with saline-HEPES medium and fixed in $2 \%$ paraformaldehyde in PBS at RT for 15 min. After fixation, the cells were washed twice with PBS and once with $\mathrm{PBS} / 1 \% \mathrm{BSA}$, permeabilised for $30 \mathrm{~min}$ using $0.1 \%$ saponin in PBS containing $1 \%$ BSA and incubated in the same buffer with $2 \mu \mathrm{l} / \mathrm{ml}$ phospho-AKT $\left(\mathrm{Ser}^{473}\right.$ ) Alexa Fluor 488 conjugate (cat no. 4071, Cell Signalling Technology) and $2 \mu \mathrm{l} / \mathrm{ml}$ phospho-AKT $\left(\mathrm{Thr}^{308}\right.$ ) Alexa Fluor 647 conjugate 
(cat no. C31E5E) for $30 \mathrm{~min}$ in the dark at $22{ }^{\circ} \mathrm{C}$. The samples were then washed in PBS. The pellets were resuspended in $500 \mu \mathrm{l}$ of PBS and analysed using a MACSQuant flow cytometer (Miltenyi Biotech, Madrid, Spain), Hoechst 33342 was added to the restrict analysis to spermatozoa and to exclude debris. The specificity of the antibodies was validated using western blotting. Negative controls consisted of unstained samples and IgG XP isotype controls (Alexa Fluor 488 and 647 conjugates).

\section{Simultaneous flow cytometric assessment of subtle membrane changes, viability and oxidative stress}

The following stock solutions were prepared in DMSO: Yo-Pro-1 $(25 \mu \mathrm{M})$, Eth-1 (1.167 mM) and CellRox (5 mM). Hoechst 33342 (1.62 mM in water) was used to identify spermatozoa and eliminate debris from the analysis. A sperm suspension $(1 \mathrm{ml})$ containing $5 \times 10^{6}$ spermatozoa $/ \mathrm{ml}$ was stained with $1 \mu \mathrm{l}$ of Yo-Pro-1, $1 \mu \mathrm{l}$ of CellRox and $0.3 \mu \mathrm{l}$ of Hoechst 33342. After thorough mixing, the sperm suspension was incubated at RT in the dark for 25 min. The spermatozoa were then washed in PBS and $0.3 \mu \mathrm{l}$ of Eth was added and incubated for $5 \mathrm{~min}$ before reading in the flow cytometer. This staining protocol was a modified version of previous protocols and distinguishes four sperm subpopulations while simultaneously measuring oxidative stress (Peña et al. 2005b, Nuñez-Martinez et al. 2007b, Ortega Ferrusola et al. 2009b). The first subpopulation, positive for only Hoechst 33342, was considered to be alive and without any membrane alterations. Another subpopulation, the Yo-Pro-1-positive cells emitting green fluorescence, showed signs of early damage and a shift to another physiological state, as membranes become slightly permeable during the first steps of damage, thus enabling Yo-Pro- 1 but not Eth to cross the plasma membrane. Neither probe enters intact cells. Finally, two subpopulations of dead spermatozoa were easily detected. These were either apoptotic (spermatozoa stained both with Yo-Pro-1 and Eth, emitting both green and red fluorescence) or necrotic (cells stained only with Eth, emitting red fluorescence). Spermatozoa exhibiting oxidative stress emit fluorescence in the far red spectrum. Representative cytograms of the assay are depicted in Fig. 1. The positive controls for oxidative stress were samples supplemented with $800 \mu \mathrm{M} \mathrm{SO} \mathrm{SO}_{4} \mathrm{Fe}$ and $200 \mu \mathrm{l}$ of $\mathrm{H}_{2} \mathrm{O}_{2}$ (Sigma) to produce the Fenton reaction.

\section{Simultaneous flow cytometric detection of active caspases 3 and 7 and active mitochondria}

CellEvent Caspase-3/7 Green Detection Reagent is a fluorogenic substrate for activated caspases 3 and 7 . The reagent consists of a four-amino-acid peptide (DEVD) conjugated to a nucleic acid-binding dye. This cell-permeant substrate is intrinsically non-fluorescent because the DEVD peptide inhibits the ability of the dye to bind to DNA. After activation of caspase- 3 and caspase- 7 in apoptotic cells, the DEVD peptide is cleaved, enabling the dye to bind to DNA and produce a bright, fluorogenic response with absorption/emission maxima of $\sim 502 / 530 \mathrm{~nm}$. One important advantage of this assay is that no wash steps are required, thus preventing cell loss during washing. Stock solutions of CellEvent (2 mM in DMSO), Eth (1.167 $\mathrm{mM}$ in DMSO), Mitotracker Deep Red $(0.5 \mu \mathrm{M}$ in DMSO) and Hoechst 33342 (1.62 mM in water) were prepared. Spermatozoa $\left(5 \times 10^{6} / \mathrm{ml}\right)$ in $1 \mathrm{ml}$ of PBS were stained with $1 \mu \mathrm{l}$ of CellEvent, $0.3 \mu$ l of Hoechst 33342 and $0.3 \mu$ l of Mitotracker

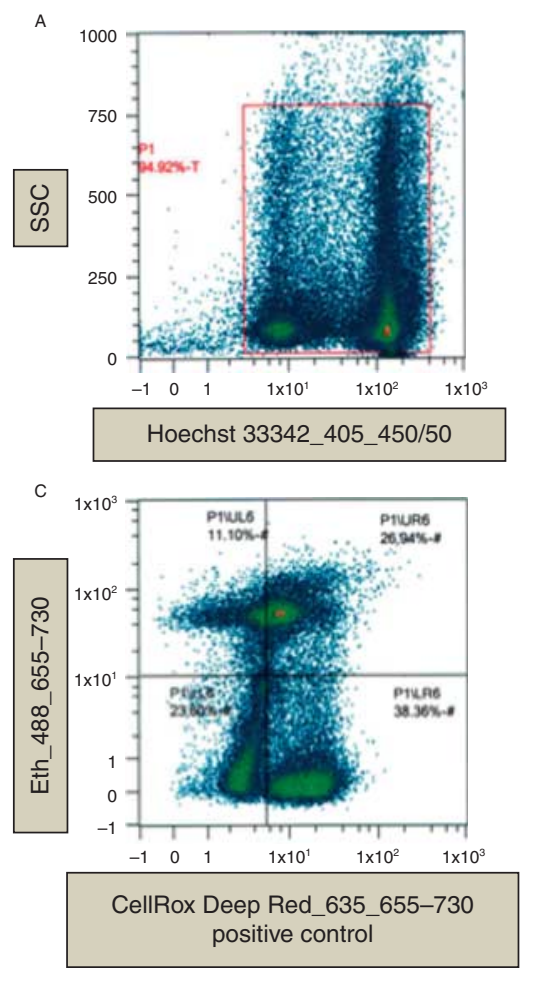

Reproduction (2014) 148 221-235
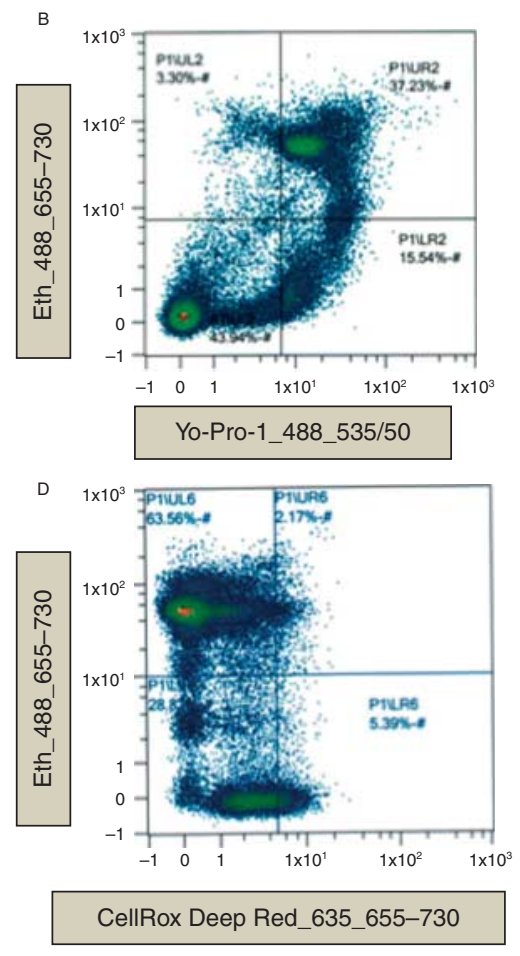

Figure 1 Representative cytograms for simultaneous flow cytometric assessment of subtle membrane changes, viability and reactive oxygen species (ROS). Samples were stained with Hoechst 33342, Yo-Pro-1, ethidium homodimer (Eth) and CellROX Deep Red, as described in the 'Materials and methods' section. Hierarchical gating was applied to exclude debris from the analysis and simultaneously measure viability, apoptosis, necrosis and ROS. (A) Hoechst 33342 fluorescence was detected using the V1 channel (Ex 405 bandpass filter 450/50 nm), and a gate was applied to positive (DNA-containing particles) events to gate out debris. The gated region was analysed. (B) Yo-Pro-1 was detected using the B1 channel (Ex 488 bandpass Filter 525/50 nm), Eth was detected using the B3 channel (Ex 488 bandpass filter 655-730 nm) and (D) CellROX Deep Red was detected using the R1 channel (Ex $635 \mathrm{~nm}$ bandpass filter $655-730 \mathrm{~nm})$. (C) Positive control for oxidative stress $\left(800 \mu \mathrm{M} \mathrm{FeSO}_{4}\right.$ plus $200 \mu \mathrm{l}$ of $\left.\mathrm{H}_{2} \mathrm{O}_{2}\right)$, $n=7$ stallions. 


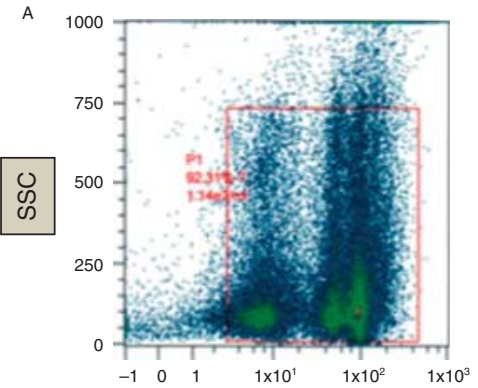

Hoechst 33342_405_450/50

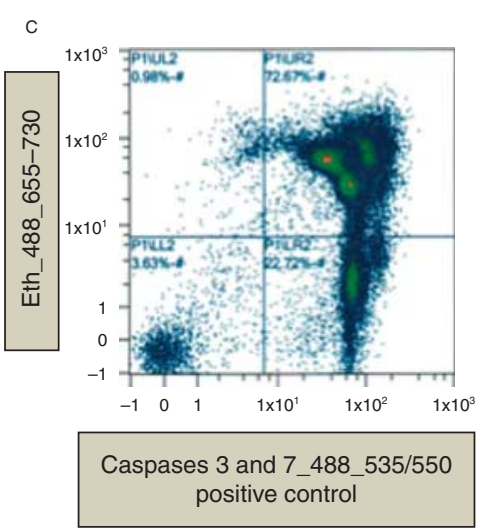

B

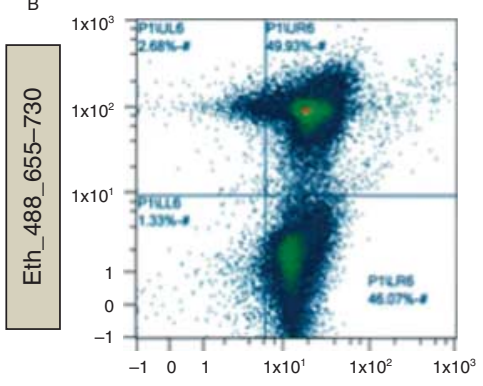

Mitotracker Deep Red_635_655-730

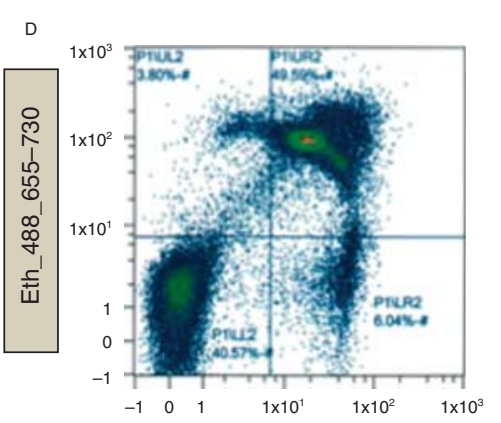

Caspases 3 and 7_488_535/550
Figure 2 Representative cytograms for simultaneous flow cytometric assessment of caspases 3 and 7 and mitochondrial activity in stallion spermatozoa. Samples were stained using CellEvent, ethidium homodimer (Eth), Mitotracker Deep Red and Hoechst 33342, as described in the 'Materials and methods' section. Hierarchical gating was applied to exclude debris from the analysis and simultaneously measure viability, caspase activity, necrosis and mitochondrial activity. (A) Hoechst 33342 fluorescence was detected using the V1 channel (Ex 405 bandPass filter 450/50 nm), and a gate was applied to positive (DNA-containing particles) events to gate out debris. The gated region was analysed. (B) Mitotracker Deep Red was detected using the R1 channel (Ex $635 \mathrm{~nm}$ bandpass filter 655-730 nm). (C) Caspases 3- and 7-positive controls (frozen thawed sperm) and (D) Caspases 3 and 7 were detected using the B1 channel (Ex 488 bandpass Filter 525/50 nm), Eth was detected using the B3 channel (Ex 488 bandpass filter $655-730 \mathrm{~nm}), n=7$ stallions.
Deep Red, and incubated in the dark at RT for $25 \mathrm{~min}$. Next, $0.3 \mu \mathrm{l}$ of Eth was added to each sample. After incubation for $5 \mathrm{~min}$, the samples were immediately run on the flow cytometer. Representative cytograms of the assay are shown in Fig. 2. Because cryopreservation induces caspase activity in stallion sperm (Ortega-Ferrusola et al. 2008), cryopreserved samples were used as positive controls (Fig. 2) for caspases 3 and 7.

\section{Evaluation of mitochondrial membrane potential $(\Delta \Psi \mathrm{m})$}

The lipophilic cationic compound JC-1 has the unique ability to differentially label mitochondria with low and high membrane potential (Peña et al. 2003b, Ortega Ferrusola et al. 2009a,b, Ortega-Ferrusola et al. c). In mitochondria with high membrane potential, JC-1 forms multimeric aggregates emitting in the high orange region $(590 \mathrm{~nm}$ ) when excited at $488 \mathrm{~nm}$. In mitochondria with low membrane potential, JC-1 forms monomers that emit in the green region $(525-530 \mathrm{~nm})$ when excited at $488 \mathrm{~nm}$. For mitochondrial membrane potential staining, a $3 \mathrm{mM}$ stock solution of JC-1 (Molecular Probes Europe) in DMSO was prepared. For each sperm sample, $1 \mathrm{ml}$ of sperm suspension in PBS $\left(5 \times 10^{6} / \mathrm{ml}\right)$ was stained with $0.5 \mu \mathrm{l}$ of JC- 1 stock solution. The samples were incubated at $37^{\circ} \mathrm{C}$ in the dark for $40 \mathrm{~min}$ before flow cytometric analysis.

\section{Determination of DNA oxidation using the 8-oxoguanine assay}

This assay is based on the direct binding of a fluorescent probe to the DNA adduct 8-oxoguanine (OxyDNA Assay Kit,
Calbiochem), which is a major oxidation product and an important indicator of free radical-induced DNA damage and oxidative stress. The assay was carried out according to the manufacturer's instructions following previously published protocols (De luliis et al. 2009, Koppers et al. 2011). In brief, spermatozoa $\left(5 \times 10^{6} / \mathrm{ml}\right)$ were separated from each sample, washed in PBS and fixed in $2 \%$ paraformaldehyde in $0.1 \mathrm{MPBS}$ $(\mathrm{pH}$ 7.6) for $15 \mathrm{~min}$ at RT. The cells were then washed twice in PBS and once in PBS containing 1\% BSA. Permeabilisation was performed for $30 \mathrm{~min}$ in PBS containing 1\% BSA supplemented with $0.1 \%$ saponin. Samples were washed with $1 \mathrm{ml}$ of wash solution (1:25 dilution in water of the wash concentrate provided by the manufacturer). Staining with $100 \mu \mathrm{l}$ of $1 \times$ FITC-Conjugate (1:10 dilution of FITC-conjugate in the wash solution) was carried out for $60 \mathrm{~min}$ in the dark at RT. Finally, the cells were washed twice and resuspended in $1 \mathrm{ml}$ of PBS for flow cytometric analysis. The amount of 8-oxiguanosine (excitation at $495 \mathrm{~nm}$ and emission at $515 \mathrm{~nm}$ ) was measured in a MACSQuant Analyser 10 (Miltenyi Biotech, Bergisch Gladbach, Germany) as green fluorescence proportional to the oxidative damage to the DNA. Positive controls were produced by incubation of additional samples in $800 \mu \mathrm{M}$ $\mathrm{Fe}^{2+}$ and $200 \mu \mathrm{M} \mathrm{H}_{2} \mathrm{O}_{2}$.

\section{MitoSox Red assay}

The generation of mitochondrial superoxide anions was investigated using MitoSox Red (MSR, Molecular Probes) as previously described (Koppers et al. 2008, 2011). Stock solutions of MitoSox Red (5 mM in DMSO) and Sytox Green $\left(0.125 \mathrm{mM}\right.$ in DMSO) were prepared. Spermatozoa $\left(5 \times 10^{6} / \mathrm{ml}\right)$ were stained with a final concentration of $2 \mu \mathrm{M}$ MitoSox Red 
and incubated for $15 \mathrm{~min}$ at $37^{\circ} \mathrm{C}$, followed by centrifugation for $5 \mathrm{~min}$ at $600 \mathrm{~g}$ and resuspension in BWW. SYTOX Green $(0.05 \mu \mathrm{M})$ was then added for a final 15 min incubation. MSR (red) and SYTOX Green (green) fluorescence were detected using 530/30 nm bandpass (green) and 585/42 nm bandpass (red) filters. Non-sperm-specific events were gated out after staining with Hoechst $33342(0.1 \mu \mathrm{l}$ of a $16 \mathrm{mM}$ solution). In total, 10000 cells were examined.

\section{TUNEL assay}

DNA cleavage may yield double- and single-stranded DNA breaks. Both types of break can be detected by labelling the free $3^{\prime}-\mathrm{OH}$ terminals with fluorescent nucleotides (labelled dUTPs) in an enzymatic reaction catalysed by TDT (Koppers et al. 2011). The samples were washed in PBS and fixed in $2 \%$ paraformaldehyde in $0.1 \mathrm{M}$ PBS ( $\mathrm{pH} 7.6$ ) for $15 \mathrm{~min}$ at RT. The cells were then washed in PBS and maintained in $5 \mathrm{ml}$ of $70 \%$ methanol at $-20{ }^{\circ} \mathrm{C}$. For recognition of DNA fragmentation in apoptotic cells, an APO-BrdU TUNEL Assay Kit (Invitrogen, Molecular Probes) was used. This kit allows detection of 3 '-hydroxyl ends that serve as starting points for TdT, which adds deoxyribonucleotides in a template-independent fashion. Addition of the deoxythymidine analogue BrdUTP to the TdT reaction labels the break sites. Once incorporated into the DNA, BrdUTP is detected by an anti-BrdUTPantibody, which is labelled by Alexa Fluor 488 (495 nm excitation and $519 \mathrm{~nm}$ emission). The total cellular DNA content is measured using propidium iodide (excitation $535 \mathrm{~nm}$ and emission $617 \mathrm{~nm}$ ). The frozen cells were washed twice in wash buffer and resuspended in the DNA-labelling solution for $60 \mathrm{~min}$ at $37^{\circ} \mathrm{C}$ in a water bath. The suspensions were washed twice in rinse buffer, and the Alexa Fluor 488-labelled anti-BrdU antibody was added to each suspension. The samples were incubated for $30 \mathrm{~min}$ at RT in the dark. Finally, $0.5 \mathrm{ml}$ of propidium iodide RNase staining buffer was added. After 30 additional minutes of incubation in the dark at RT, flow cytometry was carried out.

\section{Statistical analysis}

Three ejaculates from seven individual stallions were collected. All experiments were repeated at least three times with independent samples (three independent ejaculates from each of the seven stallions). The normality of the data was previously assessed using the Kolmogorov-Smirnoff test, in view of the normality of the distribution the results were analysed by ANOVA and the Tukey's post hoc test to perform pair wise comparisons using SPSS 19.0 Software for Mac. $P<0.05$ was regarded as significant, with $* P<0.05$ and $* * P<0.01$. Results are given as means \pm s.D.

\section{Results}

\section{Identification and subcellular localisation of phosphorylated AKT $\left(\operatorname{Ser}^{473}\right)$ and AKT $\left(\operatorname{Thr}^{308}\right)$ in stallion spermatozoa}

Phosphorylated AKT in stallion spermatozoa was detected using specific antibodies, western blotting, flow cytometry and confocal laser microscopy. Three labelling patterns were present in equine sperm, i.e. nonlabelled cells (not shown), cells labelled in the mid piece (Fig. 3) and cells labelled in the post acrosomal region (not shown). This subcellular localisation is consistent with the putative role of inhibiting pro-apoptotic proteins localised in mitochondrial membranes. Not all spermatozoa expressed phosphorylated AKT, and the staining pattern was fairly constant.

\section{Effect of SH5 on AKT phosphorylation in stallion spermatozoa}

In stallion spermatozoa, AKT was phosphorylated at $\mathrm{Thr}^{308}$ and $\mathrm{Ser}^{473}$. Inhibition of AKT phosphorylation was monitored at the beginning and after 1 and $4 \mathrm{~h}$

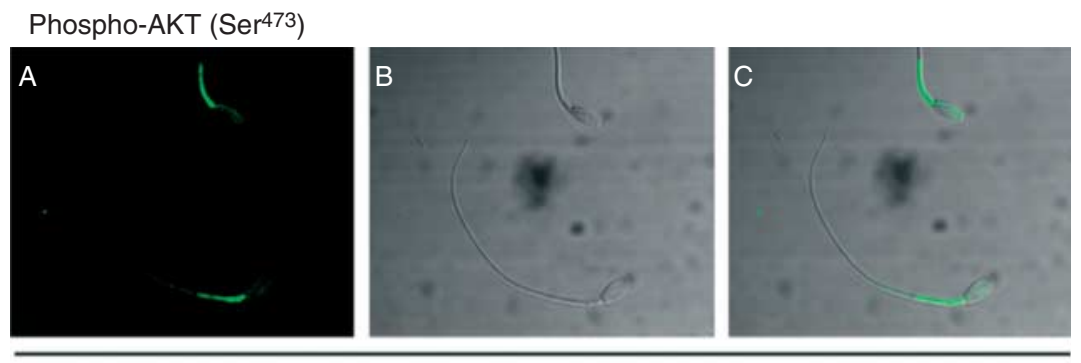

Secondary antibody
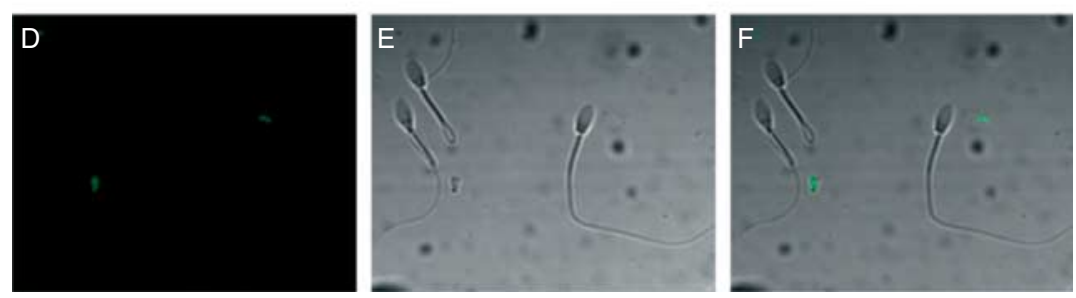

Figure 3 Subcellular localisation of phospho-AKT in stallion spermatozoa. Ejaculated stallion spermatozoa were washed and stained using specific anti-phospho-AKT antibodies and visualised using confocal laser microscopy, as described in the 'Materials and methods' section. Three labelling patterns of phosphorylated AKT were present in equine sperm, i.e. non-labelled cells (not shown), cells labelled in the mid piece (shown in this figure) and cells labelled in the post acrosomal region (not shown). (A) Transmission image, (B) fluorescence recorded using the $540 / 30 \mathrm{~nm}$ emission filter, (C) merged images, (D, E and F) negative controls, stained only with the secondary antibody, $n=7$ stallions. 

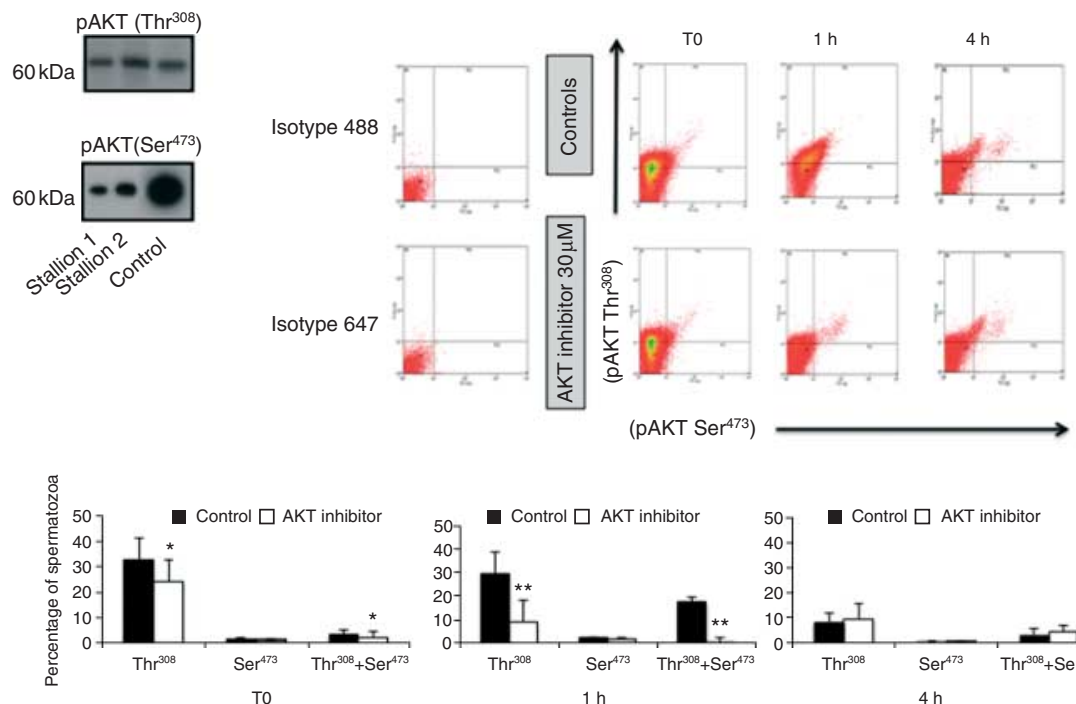

(pAKT Ser $\left.{ }^{473}\right)$
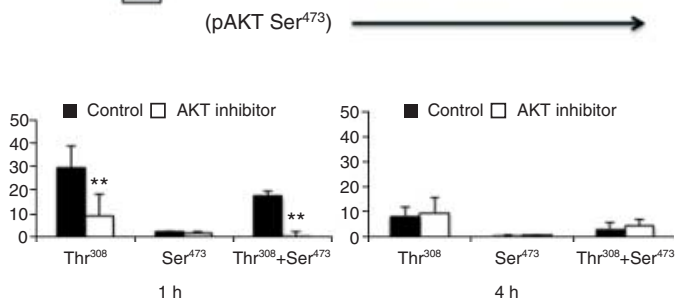

Figure 4 Effect of the AKT inhibitor SH5 on AKT phosphorylation at $\mathrm{Ser}^{473}$ and $\mathrm{Thr}^{308}$ in stallion spermatozoa. Stallion spermatozoa were washed and incubated in the presence of AKT inhibitor $(0$ or $30 \mu \mathrm{M})$ for up to $4 \mathrm{~h}$. They were then stained with specific anti-phospho-AKT antibodies (phospho-AKT $\left(\mathrm{Ser}^{473}\right)$ Alexa Fluor 488 conjugate and phospho-AKT $\left(\mathrm{Thr}^{308}\right)$ Alexa Fluor 647 conjugate, as described in the 'Materials and methods' section. Samples were analysed by flow cytometry and representative cytograms are given. Events in the lower right quadrant represent spermatozoa positive for pAKT Ser ${ }^{473}$. Events in the upper right quadrant represent spermatozoa positive for pAKT at both Ser ${ }^{473}$ and Thr ${ }^{308}$. Events in the upper left quadrant represent spermatozoa positive for pAKT Thr ${ }^{308}$. The specificity of the antibodies was validated using western blotting analysis (upper left corner), as described in the 'Materials and methods' section. Lanes one and two represent sperm lysates from two different stallions and lane three represents the positive control (pancreatic acini). In the three lower panels, the quantification of the phosphorylation status of AKT is given at three different times. Figures represent percentages of sperm in the quadrants of interest; isotype controls were used to set the quadrants as described in 'Materials and methods' section. Results are given as mean \pm s.D. Comparisons are made between each sample and the control $(* * P<0.01$ and $* P<0.05)$, $n=7$ stallions.

of incubation. After $1 \mathrm{~h}$ of incubation in presence of SH5, phosphorylated AKT decreased significantly; however, after $4 \mathrm{~h}$ of incubation, differences among control and treated samples were no longer significant (Fig. 4). The same effect was observed for the AKT1/2 inhibitor (Fig. 9D).

\section{Inhibition of AKT leads to reduced stallion sperm motility and kinematics}

After 1, 2, 4 or $6 \mathrm{~h}$ of incubation, sperm motility and velocity were determined using a CASA analysis. After $1 \mathrm{~h}$ of incubation, both AKT inhibitors (used at $30 \mu \mathrm{M}$ ) significantly decreased the percentage of total motile spermatozoa ( 30 vs $50 \%$ in controls); the percentage of progressive sperm ( 6 vs $18 \%$ in controls); and the circular velocity (39. 5 vs $63.5 \mu \mathrm{m} / \mathrm{s}$ in controls), average velocity (21.5 vs $36.7 \mu \mathrm{m} / \mathrm{s}$ in controls) and straight-line velocity $(12.5 \mathrm{vs} 21 \mu \mathrm{m} / \mathrm{s}$ in controls) $(P<0.05)$. After $2 \mathrm{~h}$ of incubation at $37^{\circ} \mathrm{C}$, the inhibitors significantly reduced the circular velocity and average velocity of the sperm cells $(P<0.05$; Figs 5 and $9 \mathrm{~A}$ and $\mathrm{B})$. These are average values of all motile sperm. These data suggest that AKT plays a role in the regulation of stallion sperm motility and velocity. After 4 and $6 \mathrm{~h}$ of incubation, all parameters for sperm motility and kinematics decreased $(P<0.01)$, regardless of the treatment.

\section{Inhibition of AKT increases sperm membrane permeability and leads to sperm death}

To determine whether AKT regulates early changes in membrane integrity and permeability, stallion spermatozoa were analysed using flow cytometry after incubation in the presence or absence of the AKT inhibitor in BWW medium for up to $6 \mathrm{~h}$ at $37^{\circ} \mathrm{C}$. After $1 \mathrm{~h}$ of incubation at $37^{\circ} \mathrm{C}$, the percentage of spermatozoa with intact membranes decreased significantly $(P<0.05)$ from $50 \%$ in controls to $38 \%$ in the presence of $30 \mu \mathrm{M}$ AKT inhibitor. Simultaneously, the percentage of spermatozoa with increased membrane permeability and the percentages of necrotic spermatozoa increased $(P<0.05)$ (Fig. 6). The effect of the inhibitor disappeared after longer incubation periods, although the percentage of dead spermatozoa was higher after $6 \mathrm{~h}$ of incubation at $37^{\circ} \mathrm{C}$ in the presence of $30 \mu \mathrm{M}$ AKT inhibitor than in the control.

\section{Yo-Pro-1 enters stallion spermatozoa through pannexin-1 channels}

In somatic cells, Yo-Pro- 1 detects early apoptotic cells by using specific channels (pannexin-1) that are open in the very early stages of apoptosis to enter cells (Elliott et al. 2009, Chekeni et al. 2010, Sandilos et al. 2012). 

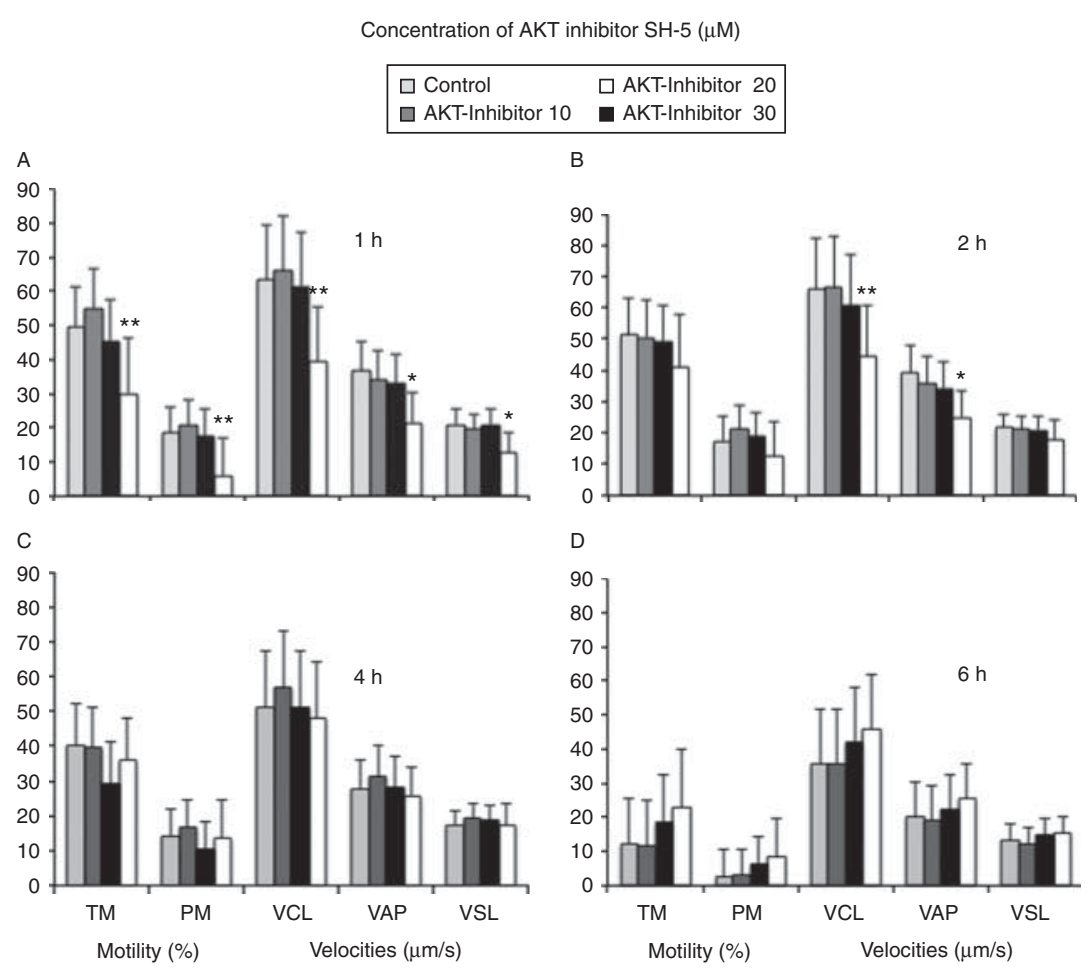

Figure 5 Effect of inhibition of AKT phosphorylation on stallion sperm motility and kinematics. Stallion spermatozoa were washed and resuspended in BWW supplemented with 1\% PVA in the presence of the AKT inhibitor SH5 at 0, 10, 20 and $30 \mu \mathrm{M}$ for up to $6 \mathrm{~h}$. (A) After $1 \mathrm{~h},(\mathrm{~B}) 2 \mathrm{~h},(\mathrm{C}) 4 \mathrm{~h}$ or (D) $6 \mathrm{~h}$ of incubation at $37^{\circ} \mathrm{C}$. TM\%, percentage of total motile spermatozoa; $\mathrm{PM} \%$, percentage of progressive motile spermatozoa; VCL $(\mu \mathrm{m} / \mathrm{s})$, circular velocity; VAP $(\mu \mathrm{m} / \mathrm{s})$, average velocity; VSL $(\mu \mathrm{m} / \mathrm{s})$, straight line velocity. Results are given as mean \pm s.D. Comparisons are made between each sample and the control $\left({ }^{* *} P<0.01\right.$ and $\left.* P<0.05\right)$, $n=7$ stallions.
To confirm Yo-Pro-1 entry through pannexin-1 channels, we first investigated pannexin-1 levels using western blotting with specific antibodies. We then blocked pannexin- 1 by incubating the spermatozoa for $1 \mathrm{~h}$ at $37^{\circ} \mathrm{C}$ in presence of two unrelated inhibitors $(1 \mathrm{mM}$ probenecid and $100 \mu \mathrm{M}$ carbenexolone) and subsequently stained only with Yo-Pro-1 in presence of Hoechst 33342 to gate out debris. Pannexin-1 was present in stallion spermatozoa, and probenecid and carbenexolone reduced the percentage of Yo-Pro-1+ spermatozoa (Fig. 7), supporting the hypothesis that Yo-Pro-1 uptake in this population of spermatozoa occurs through opening of pannexin-1 channels. Moreover, this experiment may also indicate that the stallion

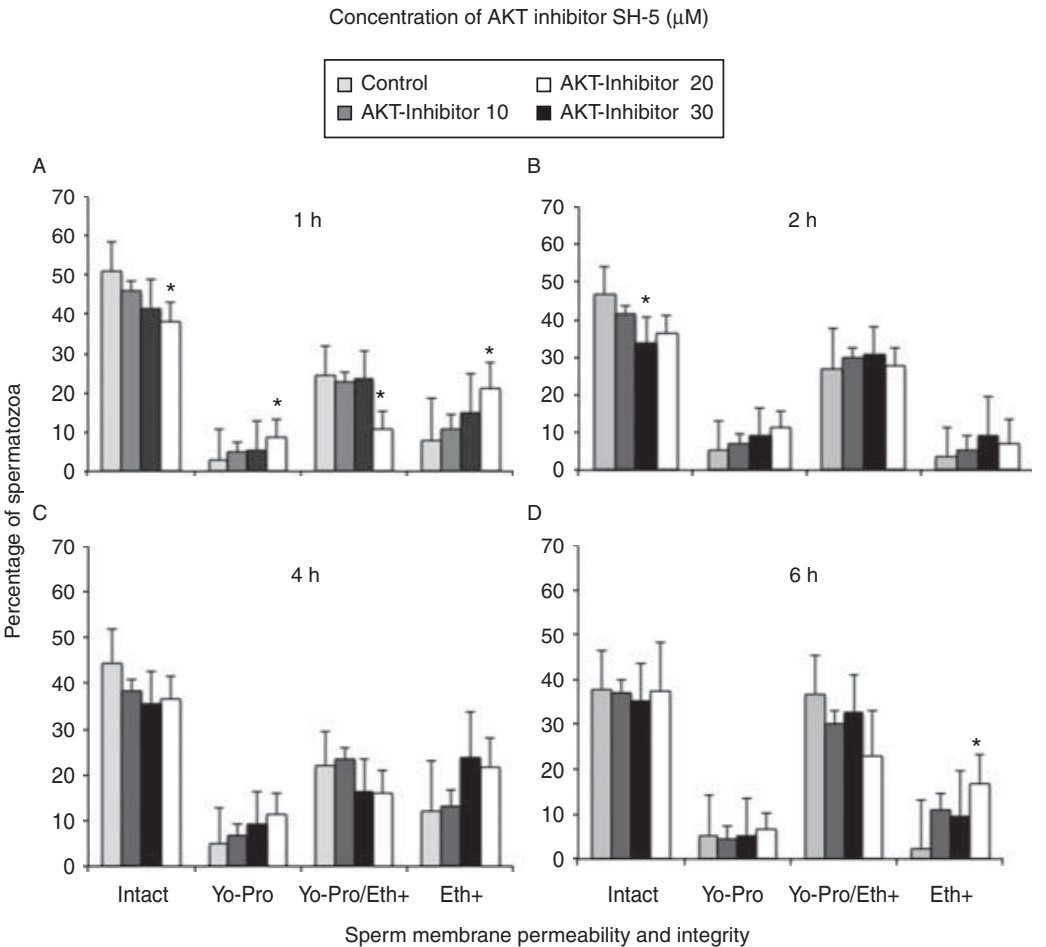

Figure 6 Effect of inhibition of AKT on stallion sperm membrane permeability and integrity. Stallion spermatozoa were washed and resuspended in BWW supplemented with $1 \%$ PVA in the presence of the AKT inhibitor $\mathrm{SH} 5$ at $0,10,20$ and $30 \mu \mathrm{M}$ for up to $6 \mathrm{~h}$. (A) After $1 \mathrm{~h},(B) 2 \mathrm{~h},(\mathrm{C}) 4 \mathrm{~h}$ and (D) $6 \mathrm{~h}$ of incubation at $37^{\circ} \mathrm{C}$. Intact \%, percentage of spermatozoa with completely intact membranes; Yo-Pro +, percentage of spermatozoa with increased permeability in their membranes; Yo-Pro+/Eth +, percentage of apoptotic spermatozoa; Eth + , percentage of dead spermatozoa. Results are given as mean \pm s.D. Comparisons are made between each sample and the control $\left({ }^{*} P<0.05\right)$, $n=7$ stallions. 


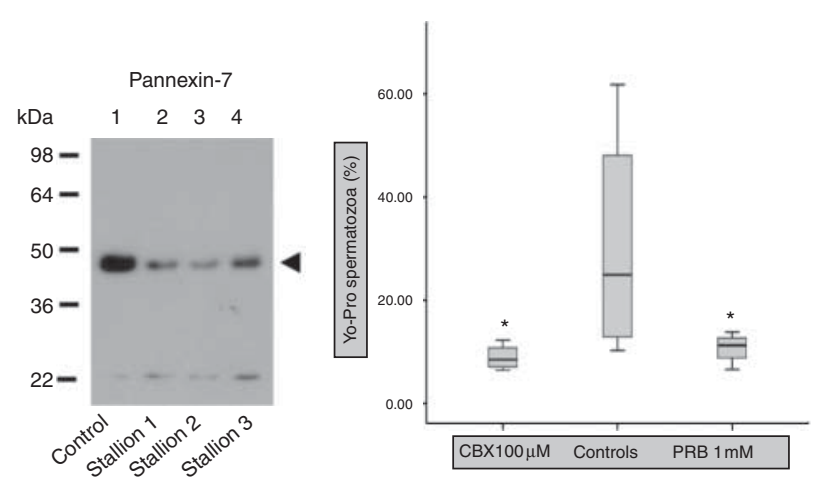

Figure 7 Identification and function of the pannexin- 1 channel in stallion spermatozoa. (Left panel) Western blot analysis showing the presence of pannexin-1 in stallion sperm lysates. Lane 1 is the control and lanes 2-4 are sperm lysates from three different stallions. (Right panel) Whisker and box plots showing the effect of two different pannexin-1 channel blockers on the uptake of Yo-Pro-1 by stallion spermatozoa. The uptake of Yo-Pro-1 was significantly inhibited by both inhibitors. Results are given as mean \pm s.D. Comparisons are made between each sample and the control $\left({ }^{*} P<0.05\right), n=7$ stallions.

spermatozoa are in the early stages of apoptotic phenomena, similar to the situation described in somatic cells (Chekeni et al. 2010).

\section{Inhibition of AKT $\left(\mathrm{Ser}^{473}\right)$ and $\left(\mathrm{Thr}^{308}\right)$ phosphorylation increases caspases 3 and 7 activity in stallion spermatozoa}

One possible mechanism that explains our findings is that the phosphorylation of AKT participates in the regulation of an apoptotic-like mechanism. To test this hypothesis, we evaluated caspases 3 and 7 activation in stallion spermatozoa after incubation in presence of the AKT inhibitor. If AKT inhibits apoptosis, its inhibition should increase caspases 3 and 7 activity. After $1 \mathrm{~h}$ of incubation, all concentrations tested reduced the percentage of caspase-negative, live spermatozoa in a positive concentration-dependent manner $(P<0.01$; Fig. 8). The percentage of caspase 3-positive spermatozoa also increased in a concentration-dependent manner and was especially evident after $4 \mathrm{~h}$ of incubation at $37^{\circ} \mathrm{C}$ (Fig. 8C). These results indicate that AKT phosphorylation at $\operatorname{Ser}^{473}$ and $\mathrm{Thr}^{308}$ inhibits activation of caspases 3 and 7 in ejaculated stallion spermatozoa. To provide further evidence supporting the role of $\mathrm{AKT}$ inhibition, an additional experiment was conducted using two different AKT inhibitors $(\mathrm{SH}-5$ and AKT1/2 kinase inhibitors at either 0 or $30 \mu \mathrm{M}$ for $1 \mathrm{~h}$ of incubation at $37^{\circ} \mathrm{C}$ ) to address the effects of AKT phosphorylation on sperm motility and velocity in addition to the activation of caspases 3 and 7 . Both inhibitors induced similar effects, causing reduced sperm motility and velocity, increase in caspases 3 and 7 activity and dephosphorylation of AKT (Fig. 9).

\section{Inhibition of $A K T$ reduces the mitochondrial membrane potential in stallion spermatozoa}

To study the effect of inhibiting AKT phosphorylation on sperm mitochondria, two different assays were carried out. The mitochondrial membrane potential was studied after JC-1 and Mitotracker Deep Red staining. Inhibition of AKT phosphorylation affected the mitochondrial membrane potential of stallion

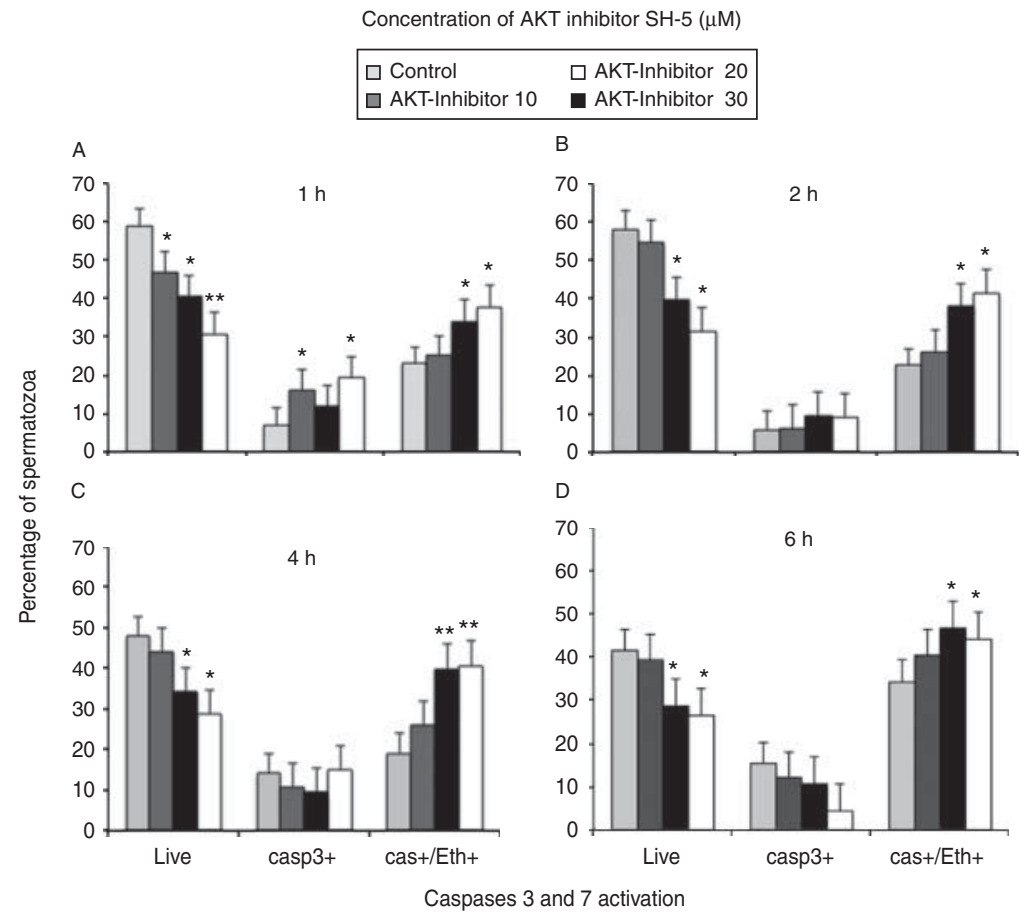

Figure 8 Effect of inhibition of AKT on caspases 3 and 7 activation in stallion spermatozoa. Samples were washed and resuspended in BWW supplemented with 1\% PVA in the presence of the AKT inhibitor SH5 at 0, 10, 20 and $30 \mu \mathrm{M}$ for up to $6 \mathrm{~h}$. (A) After $1 \mathrm{~h},(\mathrm{~B}) 2 \mathrm{~h}$, (C) $4 \mathrm{~h}$ and (D) $6 \mathrm{~h}$ of incubation at $37^{\circ} \mathrm{C}$. Live \%, percentage of live spermatozoa; casp $3+\%$, percentage of live spermatozoa with activated caspases 3 and 7 ; cas $+/$ Eth $+\%$, percentage of dead spermatozoa with activated caspases 3 and 7 . Results are given as means \pm s.D. Comparisons are made between each sample and the control ${ }^{* *} P<0.01$ and $* P<0.05), n=7$ stallions. 


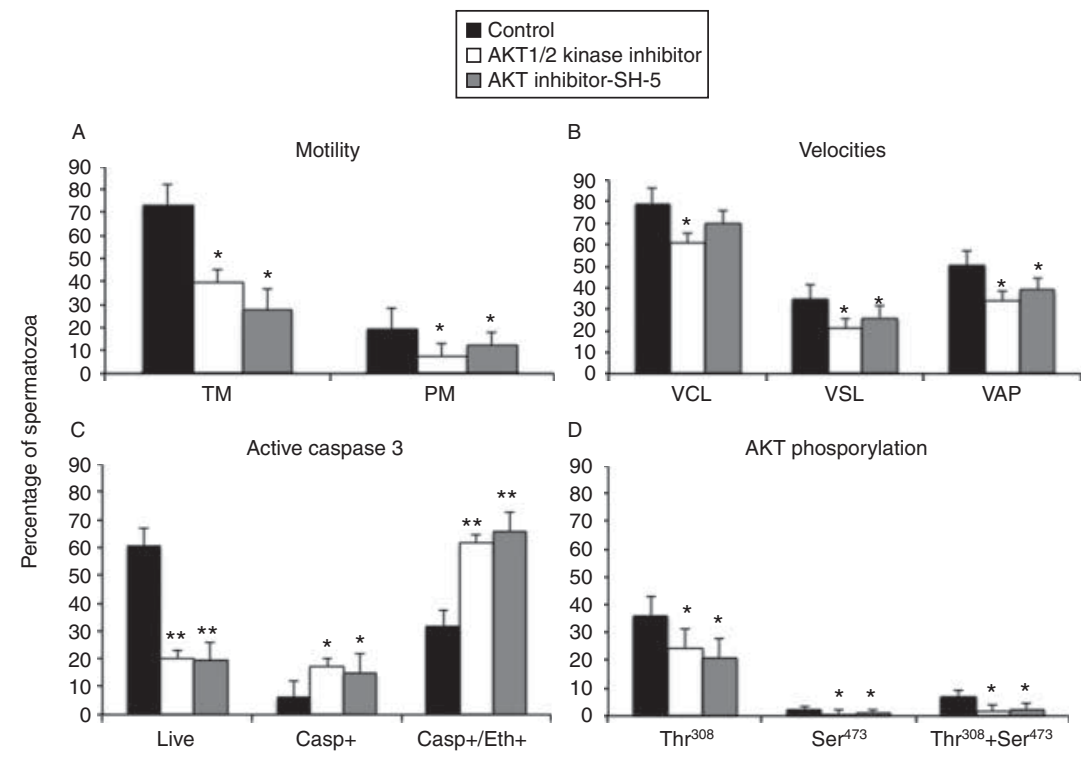

Figure 9 Effect of two different inhibitors of AKT (AKT1/2 kinase inhibitor and SH5) on motility, velocity, caspases 3 and 7 activation and AKT phosphorylation in stallion spermatozoa. The samples were washed and resuspended in BWW media and incubated for $1 \mathrm{~h}$ at $37^{\circ} \mathrm{C}$ in the presence of 0 or $30 \mu \mathrm{M}$ AKT1/2 kinase inhibitor and $30 \mu \mathrm{M} \mathrm{SH5}$. (A) Effects on stallion sperm motility. TM \%, percentage of total motile sperm; PM \%, percentage of progressive motile sperm. (B) Effects on stallion sperm velocity. VCL $(\mu \mathrm{m} / \mathrm{s})$, circular velocity; VSL ( $\mu \mathrm{m} / \mathrm{s})$, straight line velocity; VAP $(\mu \mathrm{m} / \mathrm{s})$, average velocity. (C) Effects on caspases 3 and 7 activation. Live, percentage of live sperm; Casp+, percentage of live sperm with active caspases; Casp +/Eth + , percentage of dead sperm with active caspases. (D) Effect on AKT phosphorylation. Thr ${ }^{308}$, percentage of spermatozoa with AKT phosphorylated at $\mathrm{Thr}^{308}$; $\mathrm{Ser}^{473}$, percentage of spermatozoa with AKT phosphorylated at $\mathrm{Ser}^{473}$; $\mathrm{Thr}^{308}+\mathrm{Ser}^{473}$, percentage of spermatozoa with AKT phosphorylated simultaneously at $\mathrm{Thr}^{308}$ and $\mathrm{Ser}^{473}$. Results are given as mean \pm s.D. Comparisons are made between each sample and the control $(* P<0.05$ and $* * P<0.01), n=7$ stallions.

spermatozoa in a concentration- and time-dependent manner. After $1 \mathrm{~h}$ of incubation, the AKT inhibitor at $30 \mu \mathrm{M}$ significantly reduced the mitochondrial membrane potential, as determined by both probes (Figs 10 and 11). After $2 \mathrm{~h}$ of incubation, the AKT inhibitor at 20 and $30 \mu \mathrm{M}$ decreased the mitochondrial membrane potential, as determined by Mitotracker Deep Red (Fig. 10). However, determined using JC-1, the mitochondrial membrane potential only decreased significantly at $30 \mu \mathrm{M}$ (Fig. 11).

\section{Inhibition of AKT does not increase the production of reactive oxygen species or mitochondrial superoxide, DNA oxidation or DNA fragmentation}

Because the disruption of mitochondria may lead to increased reactive oxygen species (ROS) production and oxidative stress (Koppers et al. 2008), the effect of the AKT inhibitor on apoptosis was tested. However, the differences in production of ROS were not statistically significant (Fig. 12). Furthermore, no increase in mitochondrial superoxide production was observed when AKT phosphorylation was inhibited (data not shown). Moreover, inhibition of AKT phosphorylation did not lead to significant increases in DNA oxidation (data not shown). Because DNA fragmentation is a hallmark of apoptosis, a TUNEL assay was used to evaluate apoptosis. However, inhibition of AKT did not increase the fraction of fragmented DNA (data not shown).

\section{Discussion}

The presence and the role of AKT in stallion spermatozoa were investigated using specific inhibitors. Overall, the results indicate that AKT maintains sperm survival after ejaculation through inhibition of caspases 3 and 7 . The PI3 kinase/AKT pathway has been previously studied in spermatozoa using specific inhibitors such as Wortmannin and LY294002 (du Plessis et al. 2004, Aparicio et al. 2005). However, to our knowledge, this is the first study to report the effects of specific inhibitors of AKT on mammalian spermatozoa. The data reveal a direct inhibitory effect of AKT on caspases 3 and 7, as AKT inhibition led to rapid activation of caspases 3 and 7. Thus, inhibition of an apoptosis-like mechanism (most likely being sperm-specific mechanism) plays a role in the survival of ejaculated stallion spermatozoa, which confirms similar recent findings in humans (Koppers et al. 2011) and supports the idea that postejaculatory survival of stallion and human spermatozoa is tightly regulated through the inhibition of a truncated apoptotic cascade. This inhibition is lost over time through spontaneous dephosphorylation of AKT, which suggests its function to remove redundant spermatozoa in the female genital tract (Aitken et al. 2011). However the fact that the inhibitor reduced the percentage of phospho-AKT Thr ${ }^{308}$ at the beginning of the incubation period suggests inhibition of active phosphorylation rather than dephosphorylation of this protein. 

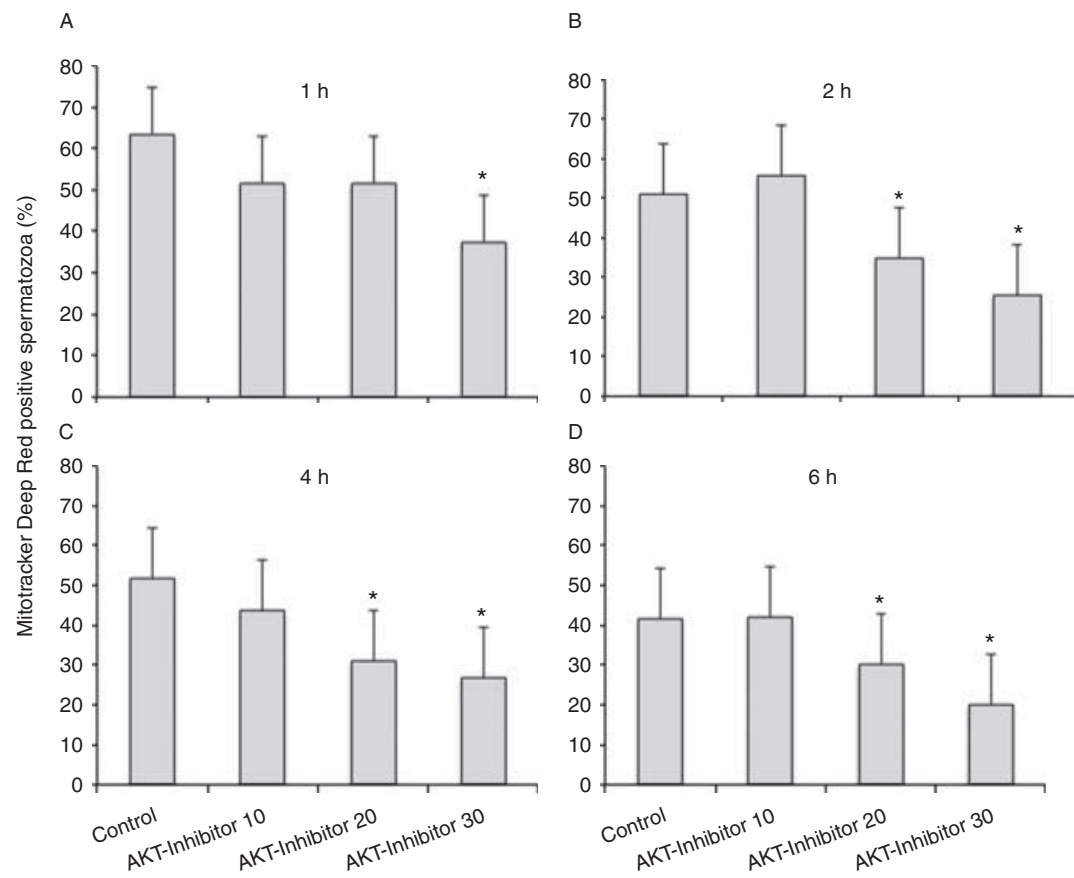

Concentration of AKT inhibitor SH-5 ( $\mu \mathrm{M})$

Figure 10 Effect of inhibition of AKT on the percentage of active mitochondria in stallion spermatozoa determined after Mitotracker staining. Samples were washed and resuspended in BWW supplemented with $1 \%$ PVA in the presence of the AKT inhibitor SH5 at $0,10,20$ and $30 \mu \mathrm{M}$ for up to $6 \mathrm{~h}$. (A) After $1 \mathrm{~h},(\mathrm{~B}) 2 \mathrm{~h},(\mathrm{C}) 4 \mathrm{~h}$ and (D) $6 \mathrm{~h}$ of incubation at $37^{\circ} \mathrm{C}$. Results are given as mean \pm S.D. Comparisons are made between each sample and the control $(* P<0.05), n=7$ stallions.

Our study suggests that AKT is necessary to maintain sperm motility, viability and mitochondrial membrane potential by inhibiting activation of caspases 3 and 7, as demonstrated by the rapid increase in caspases 3 and 7 activities upon inhibition of AKT. Inhibition of AKT was accompanied by decreases in the sperm motility and velocity, increases in sperm membrane permeability and decreases in mitochondrial membrane potential, all suggesting a role for AKT in the maintenance of sperm viability. Although we show evidence for each of these effects, the link between them remains to be determined. These findings strongly suggest that stallion

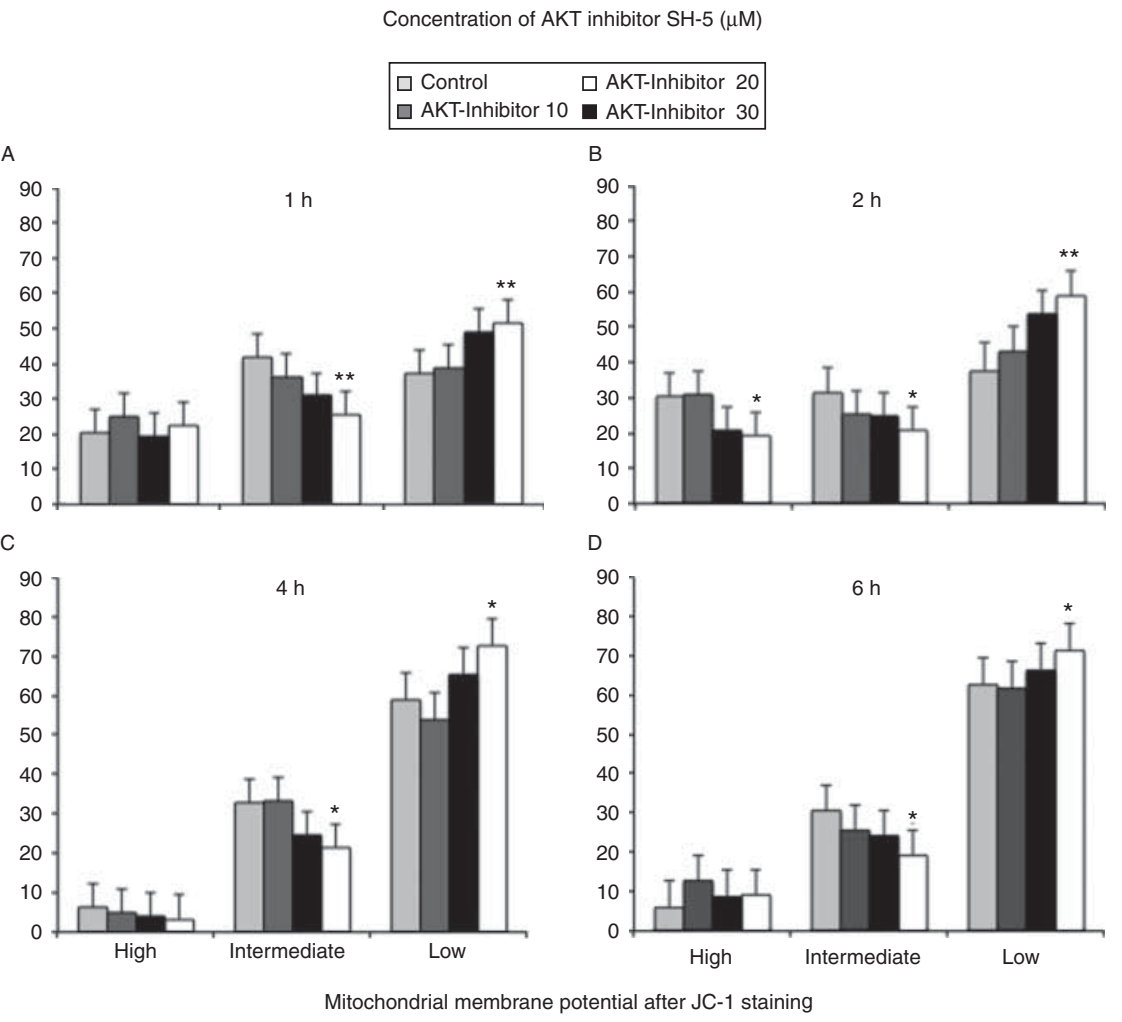

Figure 11 Effect of inhibition of AKT on the percentage of active mitochondria in stallion spermatozoa determined after JC-1 staining. Samples were washed and resuspended in BWW supplemented with 1\% PVA in the presence of the AKT inhibitor SH5 at 0, 10, 20 and $30 \mu \mathrm{M}$ for up to $6 \mathrm{~h}$. (A) After $1 \mathrm{~h}$, (B) $2 \mathrm{~h}$, (C) $4 \mathrm{~h}$ and (D) $6 \mathrm{~h}$ of incubation at $37^{\circ} \mathrm{C}$. High $\%$, percentage of spermatozoa with high mitochondrial membrane potential; intermediate $\%$, percentage of spermatozoa with mitochondria with both high and low potential; low $\%$, percentage of spermatozoa showing low mitochondrial potential mitochondrial. Results are given as mean \pm s.D. Comparisons are made between each sample and the control ${ }^{* *} P<0.01$ and $\left.* P<0.05\right), n=7$ stallions. 

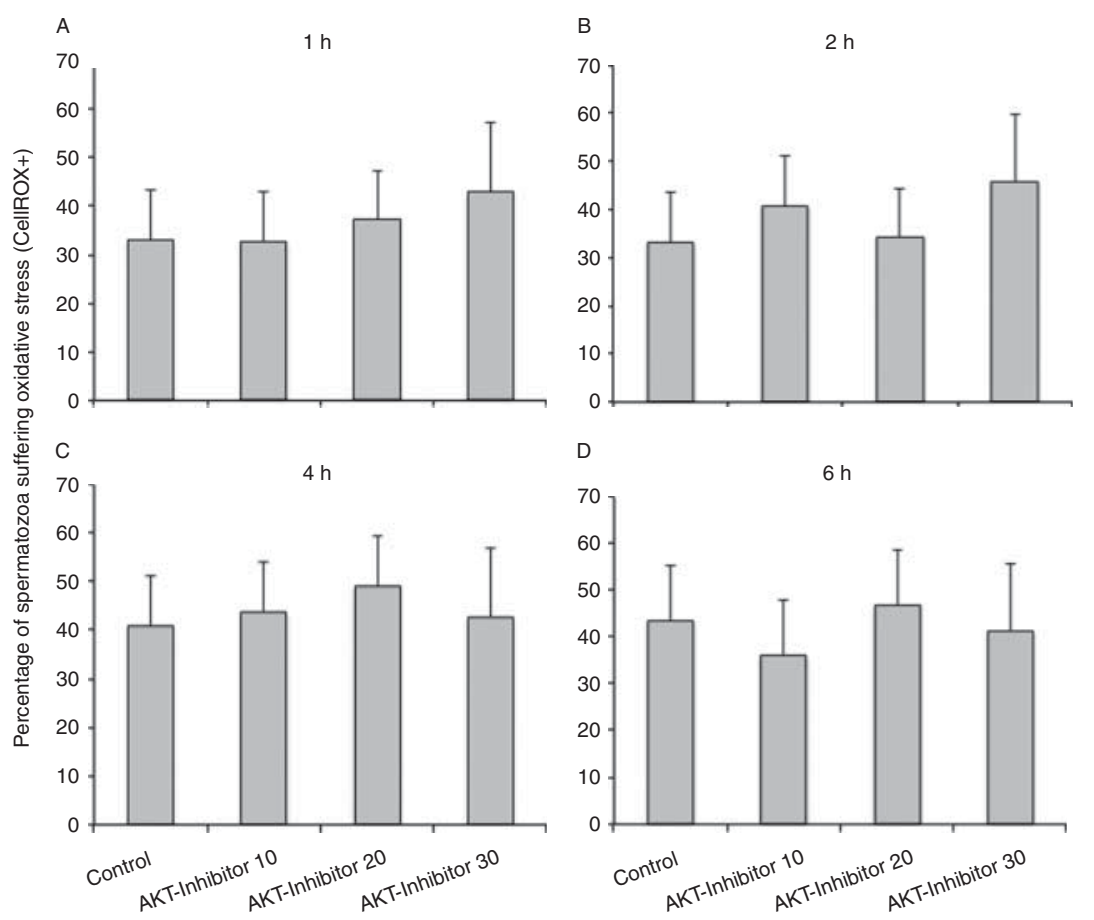

Figure 12 Effect of inhibition of AKT on the
percentage of stallion spermatozoa showing
oxidative stress. Samples were washed and resus-
pended in BWW supplemented with $1 \%$ PVA in the
presence of the AKT inhibitor SH5 at $0,10,20$ and
$30 \mu \mathrm{M}$ for up to $6 \mathrm{~h}$. (A) After $1 \mathrm{~h}$, (B) $2 \mathrm{~h},(\mathrm{C}) 4 \mathrm{~h}$
and (D) $6 \mathrm{~h}$ of incubation at $37^{\circ} \mathrm{C}$. Results are given
as mean \pm s.D. Comparisons are made between
each sample and the control, $n=7$ stallions.

sperm death during storage is largely an apoptotic phenomenon, as has been previously proposed (Ball 2008, Ortega-Ferrusola et al. 2008, da Silva et al. 2011, Macías Garcia et al. 2012). Other forms of sperm death during storage are also possible, as after $6 \mathrm{~h}$ of storage we observed an increase in the percentage of necrotic sperm incubated in the presence of $30 \mu \mathrm{M}$ $\mathrm{SH} 5$. However, most of the necrotic population was also caspase positive, which suggests an underlying apoptotic phenomenon. Moreover, when a different AKT inhibitor was used, the same effect was reproduced, which further suggests that AKT inhibition results in a truncated apoptotic pathway that causes cessation of sperm motility and death. Although we present strong evidence for the existence of this pathway, it remains to be fully described. For example, recent studies have suggested that an extrinsic pathway mediated by death receptors may also be present in spermatozoa (Macias Garcia et al. 2012, Mendoza et al. 2013).

We also demonstrated for the first time that pannexin-1 is present in stallion spermatozoa and that it has a putative role in the early stages of membrane destabilisation, as proposed for somatic cells (Elliott et al. 2009, Chekeni et al. 2010). In addition, because Yo-Pro-1 uptake occurs through pannexin-1 channels, we demonstrated the value of the Yo-Pro- 1 assay in the detection of the early stages of apoptosis. Recently, we have demonstrated a strong negative correlation between Yo-Pro-positive spermatozoa and ATP content in stallion spermatozoa (Balao da Silva et al. 2013). This finding further supports our theory, as ATP depletion in early apoptotic cells occurs through pannexin-1 channels (Elliott et al. 2009).

Apoptotic mechanisms are involved in cryodamage in mammalian spermatozoa (Ortega Ferrusola et al. 2010, Said et al. 2010). Recently, anti-apoptotic proteins have been used to improve sperm survival after thawing (Shimokawa et al. 2012). In our experiment, AKT inhibition was not accompanied by increased ROS production and DNA fragmentation, contrary to findings in humans where inhibition of PIK3CA was accompanied by increased mitochondrial production of ROS and oxidative DNA damage without changes in mitochondrial membrane potential (Koppers et al. 2011). This discrepancy could be attributed to species differences and/or to the differential effect of the inhibitor used on the PIK3CA/AKT pathway (we specifically induced inhibition of AKT downstream of PIK3CA). The inhibition of PIK3CA may also affect other downstream targets. The inhibition of PIK3CA increases cAMP levels and tyrosine phosphorylation of the PKA-anchoring protein AKAP3 in human spermatozoa, suggesting a negative role for $\mathrm{PI} 3 \mathrm{~K}$ in the regulation of motility (Luconi et al. 2001, du Plessis et al. 2004, Luconi et al. 2005). Moreover, studies using pig spermatozoa showed no effect of PIK3CA on the percentage of motile or progressive motile spermatozoa, but showed an increase in sperm velocity (Aparicio et al. 2005). In addition, both Wortmannin and LY294002 lack specificity and provoke different responses from spermatozoa (Nauc et al. 2004). A recent study (Pujianto et al. 2010) has shown more intense changes in caspase activation and loss of motility 
of human spermatozoa with an AKT inhibitor than with the PIK3CA inhibitor Wortmannin. In contrast to human sperm, stallion spermatozoa showed a decreased mitochondrial membrane potential but did not show oxidative stress upon AKT inhibition. This finding supports the existence of species-specific features in the apoptotic mechanisms of sperm senescence. Previous reports from our laboratory indicate that the mitochondrial permeability transition pore plays a role in the apoptotic mechanism associated with sperm senescence induced by cryopreservation (Ortega Ferrusola et al. 2010), which is consistent with AKT preventing translocation of Bax from the cytosol to the mitochondria.

The inhibition of AKT did not result in significant increases in DNA fragmentation, suggesting that this apoptotic mechanism does not cause DNA damage, as has previously been suggested in human spermatozoa (Aitken \& Koppers 2011, Koppers et al. 2011, Aitken et al. 2012). This lack of DNA damage can be attributed to the special architecture of spermatozoa that prevents endonucleases activated at the mid-piece during the apoptotic process from reaching the nucleus (Koppers et al. 2011). Another possible explanation arises from the relatively high resistance of stallion spermatozoa to oxidative insults relative to human sperm (Ortega Ferrusola et al. 2009a,b), a factor that most likely relates to the different lipid compositions of the sperm membranes between the species (Garcia et al. 2011, Macías Garcia et al. 2011a,b).

It is worth mentioning that multicolour flow cytometry, alongside the development of new assays for the simultaneous evaluation of several sperm characteristics and improved discrimination of sperm from debris during analysis, offers a major improvement for flow cytometric sperm assays (Petrunkina \& Harrison 2011). In conclusion, inhibition of a sperm-specific apoptotic mechanism is involved in the survival of stallion spermatozoa after ejaculation. This special form of apoptosis in spermatozoa can be termed spermptosis. Regulation of sperm survival after ejaculation is an interesting area of research with relevance to infertility in humans, particularly with respect to intrinsic sperm dysfunction and the development of reproductive technologies. In addition, stallion spermatozoa experience an apoptosis phenomenon leading to cellular senescence after ejaculation that shares biochemical and molecular characteristics with the process observed in human sperm. Thus, stallion sperm may be suitable models for studying the apoptotic mechanisms involved in male factor human infertility.

\section{Declaration of interest}

The authors declare that there is no conflict of interest that could be perceived as prejudicing the impartiality of the research reported.

\section{Funding}

The authors received financial support for this study from Ministerio de Ciencia e Innovación-FEDER, Madrid, Spain, grants AGL2013-43211-R, BFU2011-30261, Junta de Extremadura-FEDER (GR 10010 and PCE1002), VR-2011-6353 and Formas-2011-512, Stockholm, Sweden.

\section{Acknowledgements}

This paper is dedicated to the memory of Dr Fernando Peña-Martín (1922-2013).

\section{References}

Aitken RJ \& Koppers AJ 2011 Apoptosis and DNA damage in human spermatozoa. Asian Journal of Andrology 13 36-42. (doi:10.1038/aja. 2010.68)

Aitken RJ, Findlay JK, Hutt KJ \& Kerr JB 2011 Apoptosis in the germ line. Reproduction 141 139-150. (doi:10.1530/REP-10-0232)

Aitken RJ, De Iuliis GN, Gibb Z \& Baker MA 2012 The simmet lecture: new horizons on an old landscape - oxidative stress, DNA damage and apoptosis in the male germ line. Reproduction in Domestic Animals 47 (Suppl 4) 7-14. (doi:10.1111/j.1439-0531.2012.02049.x)

Aparicio IM, Gil MC, Garcia-Herreros M, Pena FJ \& Garcia-Marin LJ 2005 Inhibition of phosphatidylinositol 3-kinase modifies boar sperm motion parameters. Reproduction 129 283-289. (doi:10.1530/rep.1.00447)

Aquila S, Middea E, Catalano S, Marsico S, Lanzino M, Casaburi I, Barone I, Bruno R, Zupo S \& Ando S 2007 Human sperm express a functional androgen receptor: effects on PI3K/AKT pathway. Human Reproduction 22 2594-2605. (doi:10.1093/humrep/dem243)

Balao da Silva CM, Ortega Ferrusola C, Morillo Rodriguez A, Gallardo Bolanos JM, Plaza Davila M, Morrell JM, Rodriguez Martinez $\mathbf{H}$, Tapia JA, Aparicio IM \& Pena FJ 2013 Sex sorting increases the permeability of the membrane of stallion spermatozoa. Animal Reproduction Science 138 241-251. (doi:10.1016/j.anireprosci.2013. 02.021)

Ball BA 2008 Oxidative stress, osmotic stress and apoptosis: impacts on sperm function and preservation in the horse. Animal Reproduction Science 107 257-267. (doi:10.1016/j.anireprosci.2008.04.014)

Baumgarth N \& Roederer M 2000 A practical approach to multicolor flow cytometry for immunophenotyping. Journal of Immunological Methods 243 77-79. (doi:10.1016/S0022-1759(00)00229-5)

Busch F, Mobasheri A, Shayan P, Lueders C, Stahlmann R \& Shakibaei M 2012 Resveratrol modulates interleukin-1 $\beta$-induced phosphatidylinositol 3 -kinase and nuclear factor $\kappa B$ signaling pathways in human tenocytes. Journal of Biological Chemistry 287 38050-38063. (doi:10.1074/jbc. M112.377028)

Chekeni FB, Elliott MR, Sandilos JK, Walk SF, Kinchen JM, Lazarowski ER, Armstrong AJ, Penuela S, Laird DW, Salvesen GS et al. 2010 Pannexin 1 channels mediate 'find-me' signal release and membrane permeability during apoptosis. Nature 467 863-867. (doi:10.1038/nature09413)

De Iuliis GN, Thomson LK, Mitchell LA, Finnie JM, Koppers AJ, Hedges A, Nixon B \& Aitken RJ 2009 DNA damage in human spermatozoa is highly correlated with the efficiency of chromatin remodeling and the formation of 8-hydroxy-2'-deoxyguanosine, a marker of oxidative stress. Biology of Reproduction 81 517-524. (doi:10.1095/biolreprod.109.076836)

Elliott MR, Chekeni FB, Trampont PC, Lazarowski ER, Kadl A, Walk SF, Park D, Woodson RI, Ostankovich M, Sharma P et al. 2009 Nucleotides released by apoptotic cells act as a find-me signal to promote phagocytic clearance. Nature 461 282-286. (doi:10.1038/nature08296)

Garcia BM, Fernandez LG, Ferrusola CO, Salazar-Sandoval C, Rodriguez AM, Martinez HR, Tapia JA, Morcuende D \& Pena FJ 2011 Membrane lipids of the stallion spermatozoon in relation to sperm quality and susceptibility to lipid peroxidation. Reproduction in Domestic Animals 46 141-148. (doi:10.1111/j.1439-0531.2010. 01609.x) 
Gilot D, Giudicelli F, Lagadic-Gossmann D \& Fardel O 2010 AKTi-1/2, an allosteric inhibitor of AKT 1 and 2, efficiently inhibits CaMKI $\alpha$ activity and aryl hydrocarbon receptor pathway. Chemico-Biological Interactions 188 546-552. (doi:10.1016/j.cbi.2010.08.011)

Gonzalez-Fernandez L, Ortega-Ferrusola C, Macias-Garcia B, Salido GM, Pena FJ \& Tapia JA 2009 Identification of protein tyrosine phosphatases and dual-specificity phosphatases in mammalian spermatozoa and their role in sperm motility and protein tyrosine phosphorylation. Biology of Reproduction 80 1239-1252. (doi:10.1095/biolreprod.108.073486)

Hers I, Vincent EE \& Tavare JM 2011 AKT signalling in health and disease. Cellular Signalling 23 1515-1527. (doi:10.1016/j.cellsig.2011.05.004)

Koppers AJ, De luliis GN, Finnie JM, McLaughlin EA \& Aitken RJ 2008 Significance of mitochondrial reactive oxygen species in the generation of oxidative stress in spermatozoa. Journal of Clinical Endocrinology and Metabolism 93 3199-3207. (doi:10.1210/jc.2007-2616)

Koppers AJ, Mitchell LA, Wang P, Lin M \& Aitken RJ 2011 Phosphoinositide 3-kinase signalling pathway involvement in a truncated apoptotic cascade associated with motility loss and oxidative DNA damage in human spermatozoa. Biochemical Journal 436 687-698. (doi:10.1042/ BJ20110114)

Krutzik PO, Irish JM, Nolan GP \& Perez OD 2004 Analysis of protein phosphorylation and cellular signaling events by flow cytometry: techniques and clinical applications. Clinical Immunology $\mathbf{1 1 0}$ 206-221. (doi:10.1016/j.clim.2003.11.009)

Lan TH, Xu ZW, Wang Z, Wu YL, Wu WK \& Tan HM 2011 Ginsenoside Rb1 prevents homocysteine-induced endothelial dysfunction via PI3K/AKT activation and PKC inhibition. Biochemical Pharmacology 82 148-155. (doi:10.1016/j.bcp.2011.04.001)

Lessig J, Spalteholz H, Reibetanz U, Salavei P, Fischlechner M, Glander HJ \& Arnhold J 2007 Myeloperoxidase binds to non-vital spermatozoa on phosphatidylserine epitopes. Apoptosis 12 1803-1812. (doi:10.1007/ s10495-007-0113-5)

Luconi M, Marra F, Gandini L, Filimberti E, Lenzi A, Forti G \& Baldi E 2001 Phosphatidylinositol 3-kinase inhibition enhances human sperm motility. Human Reproduction 16 1931-1937. (doi:10.1093/humrep/ 16.9.1931)

Luconi M, Torcia S, Grillo D, Fiorenza MT, Forti G, Mangia F \& Baldi E 2005 Enhancement of mouse sperm motility by the PI3-kinase inhibitor LY294002 does not result in toxic effects on preimplantation embryo development. Human Reproduction 20 3500-3504. (doi:10.1093/ humrep/dei248)

Macías Garcia B, Gonzalez Fernandez L, Ortega Ferrusola C, Morillo Rodriguez A, Gallardo Bolanos JM, Rodriguez Martinez H, Tapia JA, Morcuende D \& Pena FJ 2011a Fatty acids and plasmalogens of the phospholipids of the sperm membranes and their relation with the postthaw quality of stallion spermatozoa. Theriogenology 75 811-818. (doi:10.1016/j.theriogenology.2010.10.021)

Macías Garcia B, Miro Moran A, Gonzalez Fernandez L, Ortega Ferrusola C, Morillo Rodriguez A, Gallardo Bolaños JM, Balao da Silva CM, Rodriguez Martinez H \& Peña FJ 2011b The mitochondria of stallion spermatozoa are more sensitive than the plasmalemma to osmotic induced stress: role of the c Jun terminal kinase (JMK) pathway. Journal of Andrology 33 105-113. (doi:10.2164/jandrol.110.011957)

Macías Garcia B, Ortega Ferrusola C, Aparicio IM, Miro-Moran A, Morillo Rodriguez A, Gallardo Bolanos JM, Gonzalez Fernandez L, Balao da Silva CM, Rodriguez Martinez H, Tapia JA et al. 2012 Toxicity of glycerol for the stallion spermatozoa: effects on membrane integrity and cytoskeleton, lipid peroxidation and mitochondrial membrane potential. Theriogenology 77 1280-1289. (doi:10.1016/j.theriogenology.2011.10.033)

Martínez-Pastor F, Jorge Tizado E, Garde JJ, Anel L \& de Paz P 2011 Opportunities and challenges of sperm motility subpopulation analysis. Theriogenology 75 783-795. (doi:10.1016/j.theriogenology.2010.11. 034)

Mendoza N, Casao A, Perez-Pe R, Cebrian-Perez JA \& Muino-Blanco T 2013 New insights into the mechanisms of ram sperm protection by seminal plasma proteins. Biology of Reproduction 88 149. (doi:10.1095/ biolreprod.112.105650)

Moran JM, Madejon L, Ortega Ferrusola C \& Peña FJ 2008 Nitric oxide induces caspase activity in boar spermatozoa. Theriogenology 70 91-96. (doi:10.1016/j.theriogenology.2008.02.010)

Nauc V, De Lamirande E, Leclerc P \& Gagnon C 2004 Inhibitors of phosphoinositide 3-kinase, LY294002 and Wortmannin, affect sperm capacitation and associated phosphorylation of proteins differently: $\mathrm{Ca}^{2+}$-dependent divergences. Journal of Andrology 25 573-585. (doi:10.1002/j.1939-4640.2004.tb02828.x)

Nuñez-Martinez I, Moran JM \& Peña FJ 2007a Identification of sperm morphometric subpopulations in the canine ejaculate: do they reflect different subpopulations in sperm chromatin integrity? Zygote 15 257-266. (doi:10.1017/S0967199407004248)

Nuñez-Martinez I, Moran JM \& Peña FJ 2007b Sperm indexes obtained using computer-assisted morphometry provide a forecast of the freezability of canine sperm. International Journal of Andrology $\mathbf{3 0}$ 182-189. (doi:10.1111/j.1365-2605.2007.00743.x)

Ortega-Ferrusola C, Sotillo-Galan Y, Varela-Fernandez E, GallardoBolanos JM, Muriel A, Gonzalez-Fernandez L, Tapia JA \& Peña FJ 2008 Detection of "apoptosis-like" changes during the cryopreservation process in equine sperm. Journal of Andrology 29 213-221. (doi:10. 2164/jandrol.107.003640)

Ortega Ferrusola C, Gonzalez Fernandez L, Macias Garcia B, SalazarSandoval C, Morillo Rodriguez A, Rodriguez Martinez H, Tapia JA \& Peña FJ 2009a Effect of cryopreservation on nitric oxide production by stallion spermatozoa. Biology of Reproduction 81 1106-1111. (doi:10. 1095/biolreprod.109.078220)

Ortega Ferrusola C, Garcia BM, Gallardo-Bolanos JM, GonzalezFernandez L, Rodriguez-Martinez H, Tapia JA \& Peña FJ 2009b Lipid peroxidation, assesed with BODIPY-C11, increases after cryopreservation of stallion spermatozoa, is stallion-dependent and is related to apoptotic-like changes. Reproduction 138 55-63. (doi:10.1530/REP-080484)

Ortega-Ferrusola C, Garcia BM, Gallardo-Bolanos JM, GonzalezFernandez L, Rodriguez-Martinez H, Tapia JA \& Peña FJ 2009c Apoptotic markers can be used to forecast the freezeability of stallion spermatozoa. Animal Reproduction Science 114 393-403. (doi:10. 1016/j.anireprosci.2008.10.005)

Ortega Ferrusola C, Gonzalez Fernandez L, Salazar Sandoval C, Macias Garcia B, Rodriguez Martinez H, Tapia JA \& Peña FJ 2010 Inhibition of the mitochondrial permeability transition pore reduces "apoptosis like" changes during cryopreservation of stallion spermatozoa. Theriogenology 74 458-465. (doi:10.1016/j.theriogenology.2010.02.029)

Parte PP, Rao P, Redij S, Lobo V, D'Souza SJ, Gajbhiye R \& Kulkarni V 2012 Sperm phosphoproteome profiling by ultra performance liquid chromatography followed by data independent analysis (LC-MS(E)) reveals altered proteomic signatures in asthenozoospermia. Journal of Proteomics 75 5861-5871. (doi:10.1016/j.jprot.2012.07.003)

Peña FJ, Johannisson A, Wallgren M \& Rodriguez-Martinez H 2003a Assessment of fresh and frozen-thawed boar semen using an Annexin- $\mathrm{V}$ assay: a new method of evaluating sperm membrane integrity. Theriogenology 60 677-689. (doi:10.1016/S0093-691X(03)00081-5)

Peña FJ, Johannisson A, Wallgren M \& Rodriguez Martinez H 2003b Antioxidant supplementation in vitro improves boar sperm motility and mitochondrial membrane potential after cryopreservation of different fractions of the ejaculate. Animal Reproduction Science 78 85-98. (doi:10.1016/S0378-4320(03)00049-6)

Peña FJ, Saravia F, Garcia-Herreros M, Nuñez-martinez I, Tapia JA, Johannisson A, Wallgren M \& Rodriguez-Martinez H 2005a Identification of sperm morphometric subpopulations in two different portions of the boar ejaculate and its relation to postthaw quality. Journal of Andrology 26 716-723. (doi:10.2164/jandrol.05030)

Peña FJ, Saravia F, Johannisson A, Walgren M \& Rodriguez-Martinez H 2005 b A new and simple method to evaluate early membrane changes in frozen-thawed boar spermatozoa. International Journal of Andrology $\mathbf{2 8}$ 107-114. (doi:10.1111/j.1365-2605.2005.00512.x)

Peña FJ, Nuñez-Martinez I \& Moran JM 2006 Semen technologies in dog breeding: an update. Reproduction in Domestic Animals 41 (Suppl 2) 21-29. (doi:10.1111/j.1439-0531.2006.00766.x)

Peña FJ, Ortega-Ferrusola C, Tapia JA \& Aparicio IM 2012 How stallion sperm age in vitro? Scenario for preservation technologies Journal of Equine Veterinary Science 32 451-454. (doi:10.1016/j.jevs. 2012.05.074)

Petrunkina AM \& Harrison RA 2011 Mathematical analysis of misestimation of cell subsets in flow cytometry: viability staining revisited. Journal of Immunological Methods 368 71-79. (doi:10.1016/j.jim.2011. 02.009) 
du Plessis SS, Franken DR, Baldi E \& Luconi M 2004 Phosphatidylinositol 3-kinase inhibition enhances human sperm motility and sperm-zona pellucida binding. International Journal of Andrology 27 19-26. (doi:10.1111/j.1365-2605.2004.00440.x)

Pujianto DA, Curry BJ \& Aitken RJ 2010 Prolactin exerts a prosurvival effect on human spermatozoa via mechanisms that involve the stimulation of AKT phosphorylation and suppression of caspase activation and capacitation. Endocrinology 151 1269-1279. (doi:10.1210/en.2009-0964)

Qiao Q, Jiang Y \& Li G 2013 Inhibition of the PI3K/AKT-NF- $\kappa B$ pathway with curcumin enhanced radiation-induced apoptosis in human Burkitt's lymphoma. Journal of Pharmacological Sciences 121 247-256. (doi:10.1254/jphs.12149FP)

Roederer M 2001 Spectral compensation for flow cytometry: visualization artifacts, limitations and caveats. Cytometry 45 194-205. (doi:10.1002/ 1097-0320(20011101)45:3 < 194::AID-CYTO1163>3.0.CO;2-C)

Said TM, Gaglani A \& Agarwal A 2010 Implication of apoptosis in sperm cryoinjury. Reproductive Biomedicine Online 21 456-462. (doi:10.1016/j.rbmo.2010.05.011)

Sakkas D, Moffatt O, Manicardi GC, Mariethoz E, Tarozzi N \& Bizzaro D 2002 Nature of DNA damage in ejaculated human spermatozoa and the possible involvement of apoptosis. Biology of Reproduction $\mathbf{6 6}$ 1061-1067. (doi:10.1095/biolreprod66.4.1061)

Sandilos JK, Chiu YH, Chekeni FB, Armstrong AJ, Walk SF, Ravichandran KS \& Bayliss DA 2012 Pannexin 1, an ATP release channel, is activated by caspase cleavage of its pore-associated C-terminal autoinhibitory region. Journal of Biological Chemistry 287 11303-11311. (doi:10.1074/jbc.M111.323378)

Schulz KR, Danna EA, Krutzik PO \& Nolan GP 2007 Single-cell phosphoprotein analysis by flow cytometry. In Current Protocols in Immunology, ch 8 , unit 8 17. Ed John E Coligan et al. Wiley Blackwell, NJ, USA

Schulz KR, Danna EA, Krutzik PO \& Nolan GP 2012 Single-cell phosphoprotein analysis by flow cytometry. In Current Protocols in Immunology, ch 8, unit 8 17, pp 11-20. Ed John E Coligan et al. Wiley Blackwell , NJ USA
Sethi G, Ahn KS, Sung B, Kunnumakkara AB, Chaturvedi MM \& Aggarwal BB $2008 \mathrm{SH}-5$, an AKT inhibitor potentiates apoptosis and inhibits invasion through the suppression of anti-apoptotic, proliferative and metastatic gene products regulated by $1 \kappa \mathrm{B} \alpha$ kinase activation. Biochemical Pharmacology 76 1404-1416. (doi:10.1016/j.bcp.2008. 05.023)

Shimokawa K, Oshiro R, Yamanaka K, Ashizawa K, Ohta S \& Tatemoto H 2012 Improvement of the post-thaw qualities of Okinawan native Agu pig sperm frozen in an extender supplemented with antiapoptotic PTD-FNK protein. Theriogenology 78 1446-1455. (doi:10.1016/ j.theriogenology.2012.06.005)

da Silva CM, Macias-Garcia B, Miro-Moran A, Gonzalez-Fernandez L, Morillo-Rodriguez A, Ortega-Ferrusola C, Gallardo-Bolanos JM, Stilwell G, Tapia JA \& Pena FJ 2011 Melatonin reduces lipid peroxidation and apoptotic-like changes in stallion spermatozoa. Journal of Pineal Research 51 172-179. (doi:10.1111/j.1600-079X.2011.00873.x)

Tazzari PL, Cappellini A, Bortul R, Ricci F, Billi AM, Tabellini G, Conte R \& Martelli AM 2002 Flow cytometric detection of total and serine 473 phosphorylated AKT. Journal of Cellular Biochemistry 86 704-715. (doi:10.1002/jcb.10262)

Yang CM, Lee IT, Lin CC, Wang CH, Cherng WJ \& Hsiao LD 2013 C-Src-dependent MAPKs/AP-1 activation is involved in TNF- $\alpha$-induced matrix metalloproteinase-9 expression in rat heart-derived $\mathrm{H} 9 \mathrm{c} 2$ cells. Biochemical Pharmacology 85 1115-1123. (doi:10.1016/j.bcp.2013. 01.013)

Received 6 May 2013

First decision 26 June 2013

Revised manuscript received 29 April 2014

Accepted 19 May 2014 